\title{
Registration-based Reconstruction of Four-dimensional Cone Beam Computed Tomography
}

\author{
Christian P. V. Christoffersen, David Hansen, Per Poulsen, and Thomas Sangild Sørensen
}

\begin{abstract}
We present a new method for reconstruction of four-dimensional (4D) cone beam computed tomography from an undersampled set of $X$-ray projections. The novelty of the proposed method lies in utilizing optical flow based registration to facilitate that each temporal phase is reconstructed from the full set of acquired projections. The reconstruction of each phase thus exhibits limited aliasing despite significant intra-phase undersampling. The method is fully self-contained. Initially an approximate 4D volume is reconstructed and an inter-phase registration based hereon. A subsequent reconstruction pass integrates the optical flow estimation in a cost function formulation in which the $X$ ray projections from all temporal phases are considered for the reconstruction of each individual phase. Quantitative and qualitative evaluations were performed through reconstruction of both a numerical phantom and a clinical dataset. The obtained reconstructions are compared to the state-of-the-art alternatives of total variation regularization and prior image constrained compressed sensing. Our studies show that the proposed method is the better overall "compromise" in the depiction of both moving and stationary anatomical structures.
\end{abstract}

Index Terms-Cone beam computed tomography (CBCT), four-dimensional (4D), reconstruction, compressed sensing, optical flow registration

\section{INTRODUCTION}

$\mathbf{C}$ ONE beam computed tomography (CBCT) has become an important imaging modality for radiotherapy treatment delivery verification [1] and for interventional radiology [2]. An X-ray flat panel detector [3] is mounted on the gantry of the linear accelerator or, in the latter scenario, on a Carm system and produces a series of X-ray images acquired at different rotation angles around the patient's feet-head axis.

By regulatory recommendation the rotation of the detector is relatively slow, i.e. in the order of one minute for a full gantry rotation during which data are acquired. A one-minute acquisition duration is unproblematic when imaging anatomical structures that remain stationary during the acquisition and a corresponding three-dimensional (3D) reconstruction is easily obtained [4]. Motion induced aliasing artifacts such as streaks and blurring are however present when imaging

C.P.V. Christoffersen and T.S. Sørensen are with the Department of Computer Science, Aarhus University, Aarhus, Denmark. Correspondence e-mail: sangild@cs.au.dk.

D. Hansen, P. Poulsen, and T.S. Sørensen are with the Department of Clinical Medicine, Aarhus University, Aarhus, Denmark.

P. Poulsen is with the Department of Oncology, Aarhus University Hospital, Aarhus, Denmark.

Copyright (c) 2013 IEEE. Personal use of this material is permitted. However, permission to use this material for any other purposes must be obtained from the IEEE by sending a request to pubs-permissions@ieee.org. structures that move during the acquisition. In the presence of motion a four-dimensional (4D) reconstruction may resolve the temporal inconsistencies in the data.

Many methods have been suggested to sort the acquired $\mathrm{X}$-ray projections into respiratory phases corresponding to a given temporal resolution under an assumption of semiperiodic respiratory motion, see e.g. references [5]-[8]. This preprocessing step exposes the fundamental reconstruction problem; After sorting the acquired projection data in a suitable number of respiratory phases (we use 10 temporal phases in this work), each phase lacks the sufficient number of projections to reconstruct the desired quality images with established techniques such as the Feldkamp, Davis, and Kress (FDK) approach [4] - even when gated adaptions (g-FDK) are utilized [9]. This led to the development of iterative reconstruction techniques utilizing total variation regularization (TV) [10] and prior image constraint compressed sensing (PICCS) [11], [12] to suppress the aliasing resulting from temporal binning. TV and PICCS vastly improve the image quality in $4 \mathrm{D}$ CBCT over FDK-based reconstructions. Even for TV and PICCS reconstructions some residual artifacts remain however; TV tends to wash out fine structures, something PICCS improves upon at the cost of some temporal blurring. We provide both qualitative and quantitative illustrations of these claims below as we compare g-FDK, TV, and PICCS to the proposed new method.

Utilizing voxel-to-voxel optical flow based inter-phase image registration we present a new reconstruction method to resolve respiratory motion in $4 \mathrm{D} \mathrm{CBCT}$ of the thorax. We demonstrate that our method is competitive in its depiction of both moving and static anatomical structures.

The method we propose differs from previous attempts at combining 4D CBCT reconstruction and optical flow estimation (or other image registration based estimates) in several aspects. First of all, in many previous methods (see e.g. [13] and references therein for examples) the temporal deformation field is estimated separately from the CBCT acquisition. In the present work we are considering solely self-contained reconstructions, i.e. we do not consider techniques based on respiratory models from external sources such as other imaging modalities or optical tracking systems.

One previously described approach that combines image registration and image reconstruction for respiratory resolved $4 \mathrm{D} \mathrm{CBCT}$ is to initially reconstruct the two extreme temporal phases, e.g. the full exhale/inhale states respectively. Next, a vector displacement field is obtained from a registration of the 
two phases. Intermediate temporal phases can subsequently be resampled based on interpolation of the displacement field [14]. More generally, optical flow registration has been utilized to interpolate volumes from sets of temporally close phases [15].

A different strategy is to 1) use an established reconstruction technique (e.g. g-FDK for CBCT) to reconstruct each temporal phase, 2) register every pair of temporal phases to each other (two-way), 3) resample every temporal phase into all other phases and output the averaged phases. This approach has been described previously for different tomographic modalities and has also been adapted to 4D CBCT [16], [17]. The basic assumptions are, firstly, a good registration can be obtained despite severe phase to phase aliasing and, secondly, that the subsequent averaging (after registration-based resampling) suppresses the aliasing without significantly impacting the spatial and temporal resolution.

Our approach is different. While we also estimate the temporal displacements based on an intermediate reconstruction, we subsequently integrate those registrations into a cost function for a final reconstruction pass. Minimizing this cost function corresponds to reconstructing each phase from a full set of projections.

In other related previous work the problems of motion estimation and volume reconstruction are solved concurrently [18]-[20]. Reference [18] even avoids the initial binning step of CBCT projections to corresponding temporal phases.

Mathematically, our method probably most closely resemble a recently published method to retrospectively reconstruct respiratory gated 2D magnetic resonance imaging (MRI) from free breathing real-time images [21]. Also, it shares some methodology with super-resolution imaging techniques [22].

\section{Methodology}

The proposed 4D CBCT reconstruction is a five step processing pipeline. Each step is briefly summarized in the following and described in further detail in a dedicated section subsequently.

1. Log-transform the acquired data and sort the resultant projections into temporal bins corresponding to the desired temporal resolution. Detailed in section II-A.

2. Reconstruct a 4D volume with low spatial resolution but full temporal resolution. Use principal component analysis on the temporal signal distribution (for each spatial location) to extract a full orthogonal set of basis functions that describe the temporal variation in the data. Detailed in section II-B.

3. Reconstruct an approximate 4D volume at full spatial and temporal resolution. Detailed in section II-C.

4. Compute a voxel by voxel optical flow registration for each pair of temporal phases. Detailed in section II-D.

5. Perform final reconstruction at the full spatial and temporal resolution. Detailed in section II-E.

An overview of the involved steps is shown in Fig. 1. The progression between the individual steps is clarified next.

\section{A. The cone beam operator, projection transform and binning}

Denote by $\mathbf{u}$ the unknown volume to be reconstructed. To be distinguished by the context, $\mathbf{u}$ is either a three-
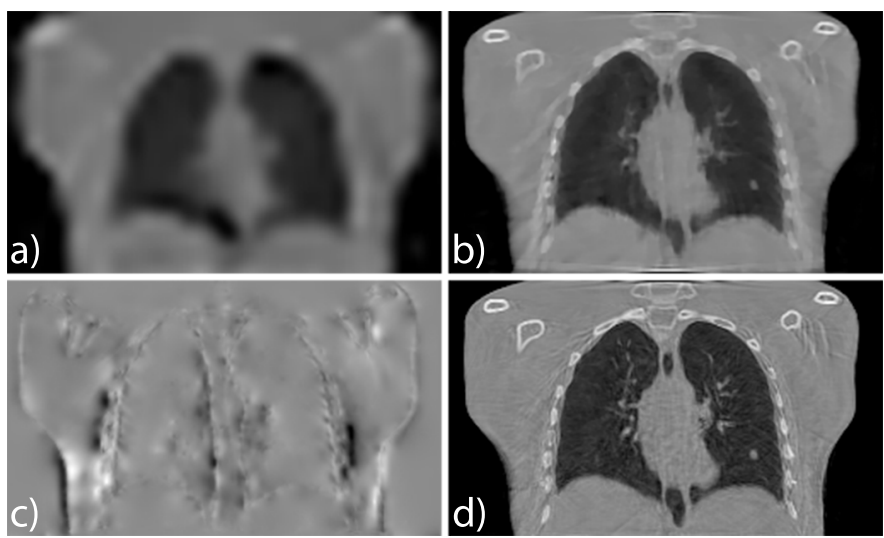

Fig. 1. Overall reconstruction pipeline for the proposed 4D CBCT reconstruction algorithm. a) After bin sorting a low spatial resolution but full temporal resolution "training dataset" is reconstructed. b) Secondly, an approximate 4D volume is produced. c) Thirdly, the inter-phase optical flow is estimated. d) Lastly, the resulting $4 \mathrm{D}$ CBCT image is reconstructed.

dimensional volume (with spatial dimensions $(x, y, z)$ ) or a four-dimensional spatio-temporal volume (with spatial dimensions $(x, y, z)$ and temporal dimension $t)$. Projection data are denoted by $\mathbf{f}$ corresponding to two spatial dimensions $\left(x^{\prime}, y^{\prime}\right)$ and a projection angle. In terms of linear algebra, $\mathbf{u}$ and $\mathbf{f}$ are both defined as column vectors, i.e. as one-dimensional vector concatenations of all elements.

The raw X-ray projection data, $\mathbf{f}^{1}$, are initially transformed element-wise to $\mathbf{f}=\ln \left(\frac{\mathbf{f}^{0}}{\mathbf{f}^{1}}\right)$ where $\mathbf{f}^{0}$ denotes a medium free calibration scan. This transform linearizes the CBCT encoding operator $\mathbf{E}$ of the unknown material attenuation coefficients $\mathbf{u}$. The acquisition data and desired reconstruction are then related through a linear system of equations:

$$
\mathbf{E u}=\mathbf{f} .
$$

By construction, each column in $\mathbf{E}$ corresponds to a unique spatio-temporal voxel in $\mathbf{u}$, and each row corresponds to a unique detector pixel/detector angle combination in $\mathbf{f}$. Thus each row in the encoding matrix $\mathbf{E}$ models a line integral (through the unknown material densities $\mathbf{u}$ ) from the X-ray source position to the corresponding detector elements in $\mathbf{f}$. For a four-dimensional volume $\mathbf{u}$ : for each row in $\mathbf{E}$, only the columns corresponding to a given temporal phase contain non-zero entries. Binning of the projection data $f$ into respiratory phases was performed using the algorithm described in references [5], [6].

As the data $\mathbf{f}$ in (1) are typically sampled such that the linear system is approximately determined in the threedimensional case (no temporal dimension), the system is severely underdetermined when the vector $\mathbf{u}$ corresponds to element concatenation of a 4D volume and the number of measurements is left unchanged. Moreover, in the presence of noise and non-linear effects such as beam hardening, no general solution to (1) exists. Instead, a linear least squares minimization problem can be cast to find

$$
\underset{\mathbf{u}}{\operatorname{argmin}}\|\mathbf{E u}-\mathbf{f}\|_{2}^{2}
$$

where $\|\cdot\|_{2}$ denotes the $l^{2}$ vector norm. 
To uniquely solve (2) a regularization term $\phi(\mathbf{u})$ is required. A solution $\hat{\mathbf{u}}$ can then be obtained from solving the constrained optimization problem

$$
\hat{\mathbf{u}}=\underset{\mathbf{u}}{\operatorname{argmin}}\{\phi(\mathbf{u})\} \quad \text { subject to }\|\mathbf{E u}-\mathbf{f}\|_{2}^{2}<\sigma^{2}
$$

where $\sigma^{2}$ indicates the tolerance level defined by the amount of noise present in the data.

This can alternatively be reformulated as an unconstrained minimization problem

$$
\hat{\mathbf{u}}=\underset{\mathbf{u}}{\operatorname{argmin}}\left\{\|\mathbf{E u}-\mathbf{f}\|_{2}^{2}+\frac{\lambda}{2} \phi(\mathbf{u})\right\} .
$$

in which $\lambda$ is the Lagrangian multiplier corresponding to a specific value of $\sigma$ in (3). In the special case of solving (4) as a purely $l^{2}$-based optimization we define $\phi(\mathbf{u})=\|\mathbf{\Gamma} \mathbf{u}-\mathbf{c}\|_{2}^{2}$ resulting in a closed form linear least squares solution

$$
\left(\mathbf{E}^{T} \mathbf{E}+\lambda \boldsymbol{\Gamma}^{T} \boldsymbol{\Gamma}\right) \hat{\mathbf{u}}=\mathbf{E}^{T} \mathbf{f}+\boldsymbol{\Gamma}^{T} \mathbf{c}
$$

which is most often solved iteratively.

\section{B. Reconstruction and principal component analysis of low- resolution training data}

Denote by $\mathbf{u}_{l}$ a spatially low resolution but full temporal resolution solution to (3) as the training data reconstruction (a name adopted from the MRI research community in which reconstruction with inherent principal component analysis (PCA) has been demonstrated previously [23], [24]). An example is provided in Fig. 1a). To suppress aliasing in $\mathbf{u}_{l}$ we minimize the spatial total variation in (3) by defining

$$
\phi(\mathbf{u})=\sum_{\mathbf{x}, t} \sqrt{\left(\nabla_{x} \mathbf{u}\right)^{2}+\left(\nabla_{y} \mathbf{u}\right)^{2}+\left(\nabla_{z} \mathbf{u}\right)^{2}}
$$

where $\nabla$ denotes the partial derivative operator (for the spatial dimension defined by the sub-script) and $\sum_{\mathbf{x}, t}$ is a sum over all spatial positions $x$ and temporal phases $t$.

Principal component analysis (PCA) was performed over the temporal variation for every spatial location in the lowresolution volume $\mathbf{u}_{l}$. This resulted in a matrix $\mathbf{A}_{l}$ of temporal basis functions, which transform $4 \mathrm{D}$ images $\hat{\mathbf{u}}$ to corresponding PCA weights $\mathbf{w}_{\hat{\mathbf{u}}}$, i.e. $\mathbf{W}_{\hat{\mathbf{u}}}=\mathbf{A}_{l} \hat{\mathbf{u}}$. Similarly to the approach proposed in reference [25], matrix $\mathbf{A}_{l}$ is carried over as a regularization term for the subsequent reconstruction pipeline step through the cost function expression $\left\|\mathbf{A}_{l} \mathbf{u}\right\|_{1}$. The overall assumption behind including the PCA term in the cost function is that the temporal dynamics do not change significantly at different scales of spatial resolution. Fig. 2 visualizes this assumption using a numerical phantom [26]. The top of Fig. 2 illustrates that for both the high-resolution ground truth image and the low resolution reconstruction most of the temporal variation is captured by the first few principal basis functions; when using just 3 basis functions at least $98 \%$ of the temporal variation can be accounted for. The bottom part of the figure illustrates the degree to which the three principal basis function agree between the high-resolution ground truth image and the low resolution reconstruction. It is clear from the figure that the two most important PCA basis functions resemble each other closely in

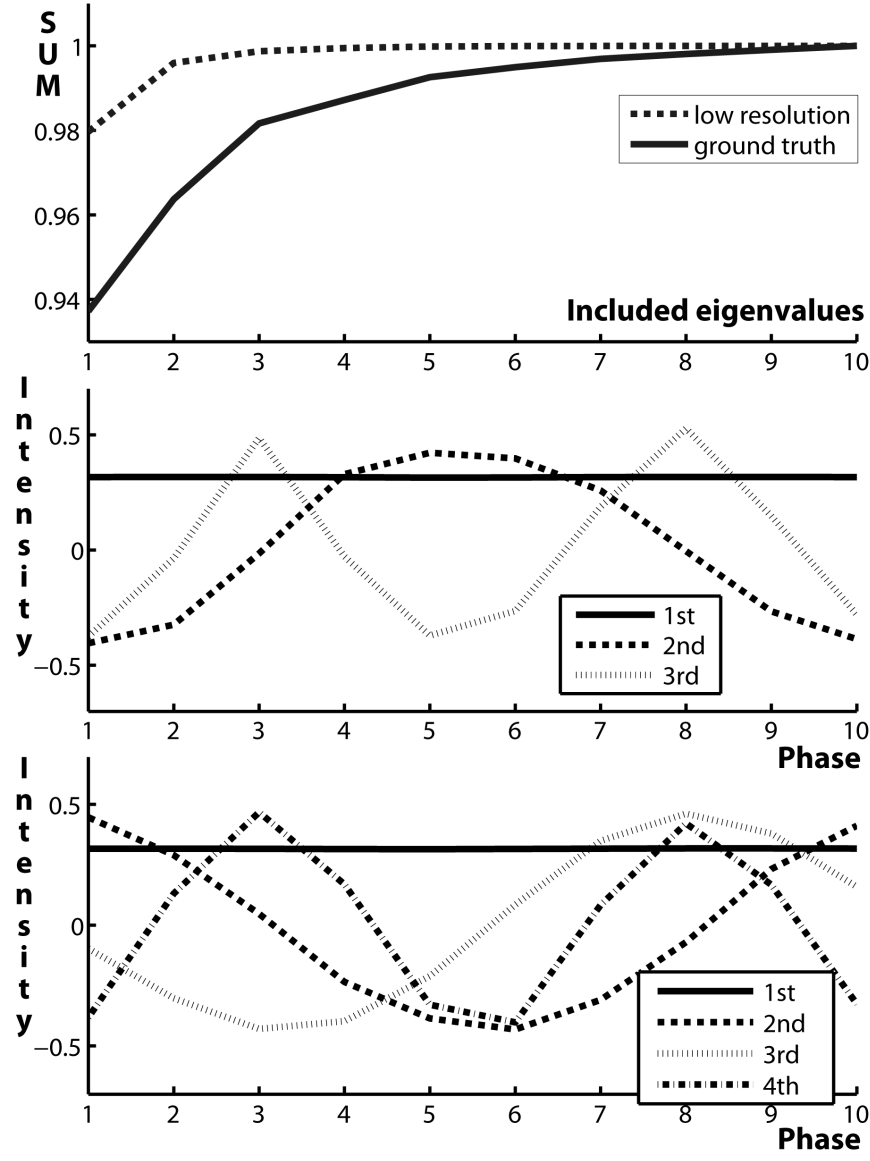

Fig. 2. Principal component analysis over the temporal dimension (10 phases) of a high-resolution numerical phantom (ground truth) and a low resolution reconstruction hereof. Top: plots of the relative cumulative sum of the 10 eigenvalues corresponding to the covariance matrix of data observations. The cumulative sum is plotted for both the ground truth and low resolution approximation. Middle: depiction of the coefficients of the most significant PCA basis functions for the ground truth dataset. Bottom: depiction of the coefficients of the most significant PCA basis functions for the low resolution reconstruction.

the two reconstructions, whereas the fourth PCA basis function for the low-resolution reconstruction resemble the third PCA basis function of the ground truth closely (disregarding the sign of the functions).

In the present work a Split-Bregman solver [27] including a non-negativity constraint [28] was utilized to minimize (3). Appendix A contains pseudo-code for the Split-Bregman solver and a brief presentation of the algorithm (Algorithm 1). Its computational core consists of repeatedly minimizing an $l^{2}$ based optimization problem in the form of (4), hence solving linear systems in the form of (5) in which the right hand side is modified for every invocation. This happens in the first line of the loop in Algorithm 1. A conjugate gradient solver, for which pseudo-code is provided in Algorithm 2, Appendix A, was utilized to solve this linear system of equations.

\section{Intermediate reconstruction for temporal registration}

The objective of the present step is a four-dimensional reconstruction, $\mathbf{u}_{r}$, of sufficient quality that volume to volume temporal registration (every volume to all other volumes) can 
subsequently be performed. Aliasing, which impacts most (if not all) registration algorithms negatively, is reduced at this stage by reconstructing $\mathbf{u}_{r}$ subject to $l^{1}$-minimization (compressed sensing [29]), i.e.

$$
\begin{aligned}
\mathbf{u}_{r}=\underset{\mathbf{u}}{\operatorname{argmin}}\left\{\|\mathbf{u}\|_{T V}+\lambda\right. & \left.\left\|\mathbf{A}_{l} \mathbf{u}\right\|_{1}\right\} \\
& \text { subject to }\|\mathbf{E u}-\mathbf{f}\|_{2}^{2}<\sigma^{2}
\end{aligned}
$$

where the total variation regularization is now applied both spatially and temporally:

$$
\begin{aligned}
& \phi(\mathbf{u})=\|\mathbf{u}\|_{T V}= \\
& \sum_{\mathbf{x}, t} \sqrt{\left(\nabla_{x} \mathbf{u}\right)^{2}+\left(\nabla_{y} \mathbf{u}\right)^{2}+\left(\nabla_{z} \mathbf{u}\right)^{2}+\left(\nabla_{t} \mathbf{u}\right)^{2}} .
\end{aligned}
$$

An example is provided in Fig. 1b). As for the preceding reconstruction of the low resolution $4 \mathrm{D}$ volume $\mathbf{u}_{l}$, a SplitBregman solver with a non-negativity constraint was used to find the solution to (7) and a conjugate gradient algorithm was used to solve the linear inner system (Algorithms 1-2, Appendix A).

By including the PCA matrix term in (7) the Split-Bregman solver converges significantly faster as it would otherwise. In all our examples it converged when running just a single conjugate gradient iteration for every Split-Bregman iteration $(N=1$ in Algorithm 2). This accelerated convergence property is our main motivation for introducing the PCA term in the present reconstruction step. The cost per Split-Bregman iteration of adding the term $\left\|\mathbf{A}_{l} \mathbf{u}\right\|_{1}$ is modest as it merely adds the regularization matrix $\mathbf{A}_{l}^{T} \mathbf{A}_{l}=\mathbf{I}$ to the conjugate gradient solver's system matrix (symbolized by $\boldsymbol{\Gamma}^{T} \boldsymbol{\Gamma}$ in Algorithm 2). However, an instance of $\mathbf{A}_{l} \mathbf{u}_{k}$ and $\mathbf{A}_{l}^{T} \mathbf{f}_{k}$ is computed for every iteration of the Split-Bregman solver. The noise suppressed and intensity homogeneous intermediate volume is well suited for subsequent optical flow based volume to volume registration over the temporal dimension.

\section{Optical flow registration}

We utilize a method proposed by Cornelius and Kanade [30], an adaption of the more well-known method by Horn and Schunck [31]. It computes the voxel-per-voxel displacement field $\mathbf{v}_{t \rightarrow t^{\prime}}$ from volume $t$ to volume $t^{\prime}$ such that $\mathbf{u}_{\mathbf{x}+\mathbf{v}_{t \rightarrow t^{\prime}}, t} \approx$ $\mathbf{u}_{\mathbf{x}, t+1}$ using a formulation based on variational calculus. Appendix B contains a brief summary of the mathematics behind the method.

Corresponding to displacement field $\mathbf{v}_{t \rightarrow t^{\prime}}$, which maps temporal volume $t$ to temporal volume $t^{\prime}$, we define the resampling matrix $\mathbf{R}_{t \rightarrow t^{\prime}}$ such that $\mathbf{R}_{t \rightarrow t^{\prime}} \mathbf{u}_{t}=\mathbf{u}_{t^{\prime}}$. This is done for all pairs of temporal phases. These matrices are the key ingredient in the final reconstruction step described below - and the main contribution of this paper.

References [30], [31] derive a linear system of equations to determine $\mathbf{v}_{t \rightarrow t^{\prime}}$. In our implementation we define a multiresolution solution; during upsampling the source image at the new resolution level is resampled based on the displacement estimates computed at the lower resolution level [32]. This results in a non-linear registration solver that was previously validated successfully for both conventional and cone beam tomography [33]. Pseudo-code can be found in Algorithm 3, Appendix B along with a brief description of the implementation. The pseudo-code denotes volume $\mathbf{u}_{t}$ as the moving image $M$ and volume $\mathbf{u}_{t+1}$ as the fixed image $F$. A visual depiction of a registration result is shown in Fig. 1c); it depicts the right-left optical flow component of a registration of the given imaging plane to a neighboring temporal phase.

\section{E. Four-dimensional reconstruction using inter-phase regis- tration}

The final step in the reconstruction process utilizes the obtained registration matrices from the previous step to compute a high spatial resolution, high temporal resolution 4D reconstruction. For simplicity, we describe the reconstruction of a single 3D temporal phase $t$ in the following. The process is repeated for the remaining phases to obtain the full $4 \mathrm{D}$ reconstruction.

Denote by $\mathbf{R}_{t}$ the block matrix formed by assembling matrices $\mathbf{R}_{t \rightarrow t^{\prime}}, \forall t^{\prime} \neq t$ in one matrix that resamples the threedimensional volume $\mathbf{u}_{t}$ to a $4 \mathrm{D}$ volume of all other phases. The purpose of the resampling matrix is to allow use of all acquired data for each phase's reconstruction. In other words, the basic reconstruction problem (1) can be formulated such that it is no longer underdetermined - even when the temporal dimension is included in $\mathbf{u}$. We formulate an unconstrained optimization problem for the final reconstruction task:

$$
\hat{\mathbf{u}}_{t}=\underset{\mathbf{u}_{t}}{\operatorname{argmin}}\left\{\left\|\mathbf{E}_{t} \mathbf{u}_{t}-\mathbf{f}_{t}\right\|_{2}^{2}+\frac{\lambda_{t^{\prime}}}{2}\left\|\mathbf{E}_{t^{\prime}} \mathbf{R}_{t} \mathbf{u}_{t}-\mathbf{f}_{t^{\prime}}\right\|_{2}^{2}\right\}
$$

In (9) subscript ${ }_{t}$ denotes temporal phase $t$ and subscript $t_{t^{\prime}}$ the remaining phases. Thus $\mathbf{E}_{t}$ and $\mathbf{f}_{t}$ denote the 3D CBCT encoding operator and data corresponding to phase $t$ while $\mathbf{E}_{t^{\prime}}$ and $\mathbf{f}_{t^{\prime}}$ denote a 4D CBCT encoding operator for phases $t_{t^{\prime}}$ and corresponding data. By $\lambda_{t^{\prime}}$ we indicate that individual weights could be applied for each temporal phase in the regularization term individually.

The main insight to read from (9) is that through registration based resampling of the unknown three-dimensional volume to the remaining temporal phases we are able to utilize all the acquired data for every three-dimensional reconstruction and thus be approximately fully sampled. An initial example of the obtained image quality can be seen in Fig. 1d).

A gradient projection solver including a non-negativity constraint [34], [35] was used to minimize (9). Appendix C contains pseudo-code (Algorithm 4) and a brief summary of the implementation.

\section{F. Material}

The proposed reconstruction method was evaluated using 1) the numerical XCAT phantom [26], and 2) a clinical dataset from a 77 years old female patient receiving radiation therapy treatment for non-small cell lung cancer.

XCAT simulation. A spherical lesion (lung tumor) of 20 $\mathrm{mm}$ diameter was placed in the right lung and a lesion of $10 \mathrm{~mm}$ diameter in the left lung. Both tumors underwent respiratory motion with four second periods and $10 \mathrm{~mm}$ peakto-peak amplitude in the feet-head direction. The acquisition 
of $680 \mathrm{X}$-ray projections was simulated in a one-minute fullfan gantry revolution. We emphasize that the simulated CBCT projections were generated by the XCAT software package using a different forwards operator than our reconstruction encoding matrix $E$. That is, the XCAT projection simulation model is completely decoupled from the subsequent reconstruction. Neither cardiac motion nor noise was simulated. The projections were temporally binned according to their relative time of acquisition within the respiratory cycle (phase binning). The resolution of the simulated digital flat panel was set to $512 \times 256$ pixels of $1.552^{2} \mathrm{~mm}^{2}$. The ground truth image and all reconstructions (apart from the initial lowresolution reconstruction) had an isotropic target resolution of $1.75^{3} \mathrm{~mm}^{3}$ corresponding to a coverage of $448 \times 336 \times 336$ $\mathrm{mm}^{3}$ on a $256 \times 192 \times 192$ matrix. The ground truth image was generated with sub-pixel division, one volume corresponding to each projection, and all volumes averaged within each temporal bin.

Clinical dataset. The clinical dataset consisted of $672 \mathrm{X}$ ray projections acquired during a $360^{\circ}$ gantry rotation by the on-board imager of a Trilogy linear accelerator (Varian Medical Systems, Palo Alto, Ca) in half-fan mode, i.e. with the projection plate offset $14.8 \mathrm{~cm}$ in the lateral direction in order to obtain an increased field of view. A single lung tumor of approximately $2 \times 4 \times 4 \mathrm{~cm}^{3}$ in size was present. The projections were temporally binned to 10 phases based on the diaphragm position using the method described in references [5], [6]. The resolution of the digital flat panel was downsampled to $512 \times 384$ pixels of $0.776^{2} \mathrm{~mm}^{2}$. The isotropic reconstruction target resolution was set to $1.75^{3} \mathrm{~mm}^{3}$ corresponding to a coverage of $448 \times 448 \times 280 \mathrm{~mm}^{3}$ on a $256 \times 256 \times 160$ matrix. A physical bowtie filter was used during the acquisition.

\section{G. Implementation}

Our reconstruction results were compared to the alternatives of g-FDK, TV, and PICCS (see the results section). The gFDK algorithm was implemented with a ramp (Ram-Lak) filter according to the outline in references [9], [36]. The TV reconstruction was implemented as the solution to the constrained optimization problem (3) with spatial and temporal TV regularization defined according to (8). The Split-Bregman solver was applied as described in Appendix A. A 3D FDK reconstruction [36] was used as the average image for the PICCS implementation, which again was based on the SplitBregman solver. For PICCS, the weight of the prior image was set to $\alpha=0.5$ in accordance with [12]. To ensure full visual convergence of the TV and PICCS solvers, they were run for 300 Split-Bregman iterations each containing 8 conjugate gradient iterations $(N=300$ in Algorithm $1, N=8$ in Algorithm 2, Appendix A).

For the registration-based reconstructions the following settings were used. For the low-resolution reconstructions the projection plate was downsampled by a factor of 16 in each direction and reconstructed into temporal volumes of $32^{3}$ voxels. One hundred Split-Bregman iterations were run with 3 conjugate gradient iterations each $(N=300$ in Algorithm 1 ,
$N=3$ in Algorithm 2, Appendix A). The PCA analysis was performed in Matlab using the 'princomp' function. The intermediate TV reconstruction was performed on a projection plate downsampled by a factor of two in each direction. One hundred Split-Bregman iterations were run with one conjugate gradient iteration each $(N=100$ in Algorithm $1, N=1$ in Algorithm 2, Appendix A). For the optical flow registration (Algorithm 3, Appendix B) we adapted GPU-based code from a previous project [33]. For the final reconstruction 50 iterations of the gradient projection solver was run $(N=50$ in Algorithm 4, Appendix C).

We utilized the open source medical image reconstruction framework Gadgetron [37] for all reconstructions. A dedicated GPU-based cone beam projection operator was written for the Gadgetron interface, basically implementing two functions providing multiplication with $\mathbf{E}$ and $\mathbf{E}^{T}$ respectively. These forwards and backwards projection operators were implemented using uniformly distributed sample points along straight integral lines. Sub-voxel distance between consecutive samples in the image space voxel map was applied. The projection operators were implemented as unmatched operators, i.e. implementing the forward operator as ray-driven and the backprojection voxel-driven as described in [38].

Host memory was used to store all vectors as the amount of memory required to store the four-dimensional image volumes, the projection data, the registration vector fields, and internal copies hereof in the respective solvers exceeds that available on present generation GPUs. Every GPU-based operator is consequently responsible for up-/downloading the required memory between the CPU and GPU at every use. This extensive transfer of data over the PCI-express bus remains one of the main performance bottlenecks in the implementation. To alleviate this somewhat, the resampling operators $\mathbf{R}_{t}$ and PCA matrices $\mathbf{A}_{l}$ were implemented on the host using the Eigen $\mathrm{C}++$ template library for linear algebra. The programming language Cuda was used for all GPU code in the project.

All reconstructions were performed on a standard Linuxbased workstation with a quad-core Intel Xeon $3.07 \mathrm{GHz}$ processor, 24 GB of host memory, and an Nvidia Geforce GTX 480 GPU with 1536 MB of device memory.

\section{RESULTS}

In the following we denote the proposed optical flow based "registration reconstruction" with the acronym OF (for optical flow). Initially a qualitative comparison between g-FDK, TV, PICCS, and OF was performed based on the numerical XCAT phantom [26]. Fig. 3 offers a side-by-side comparison of a selected coronal and sagittal slice from a single temporal phase. It is immediately apparent that g-FDK performs inadequately on insufficiently sampled data. This comes as no surprise as exactly this conclusion motivated the development of TV and PICCS.

We zoom in on two anatomical structures in motion during the respiratory cycle, i.e. the larger of the two tumors and a part of the sternum (defined by the two leftmost boxes at the top of Fig 3). Their motion throughout the 10 respiratory phases is depicted in Fig. 4 and Fig. 5. In the close-up of the tumor 

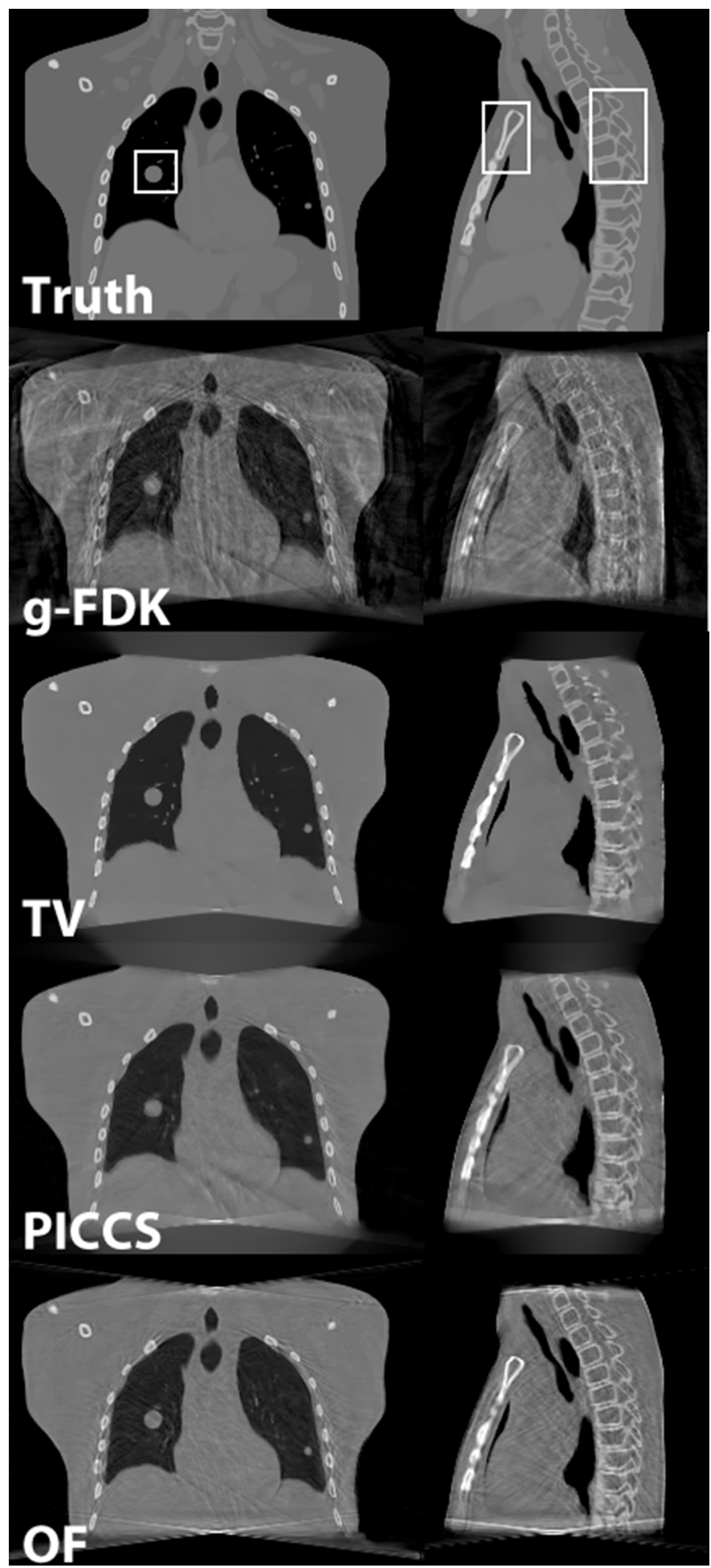

Fig. 3. Isotropic resolution reconstruction $\left(1.75^{3} \mathrm{~mm}^{3}, 10\right.$ temporal phases $)$ of the 4D numerical XCAT phantom based on simulated projections from one gantry rotation. A temporal phase with the diaphragm in significant motion was selected for illustration purposes. Comparison of optical flow based reconstruction (OF) to a gated FDK (g-FDK) reconstruction, total variation regularization (TV), and prior image constraint compressed sensing (PICCS). In the coronal view (left) a $20 \mathrm{~mm}$ in diameter spherical "tumor" is depicted in the right lung (to the left) and a $10 \mathrm{~mm}$ spherical tumor in the left lung (to the right). The rightmost column depicts a sagittal slice. Three white rectangles in the ground truth images specify the anatomical areas enlarged in Fig. 4, Fig. 5, and Fig. 6 respectively.
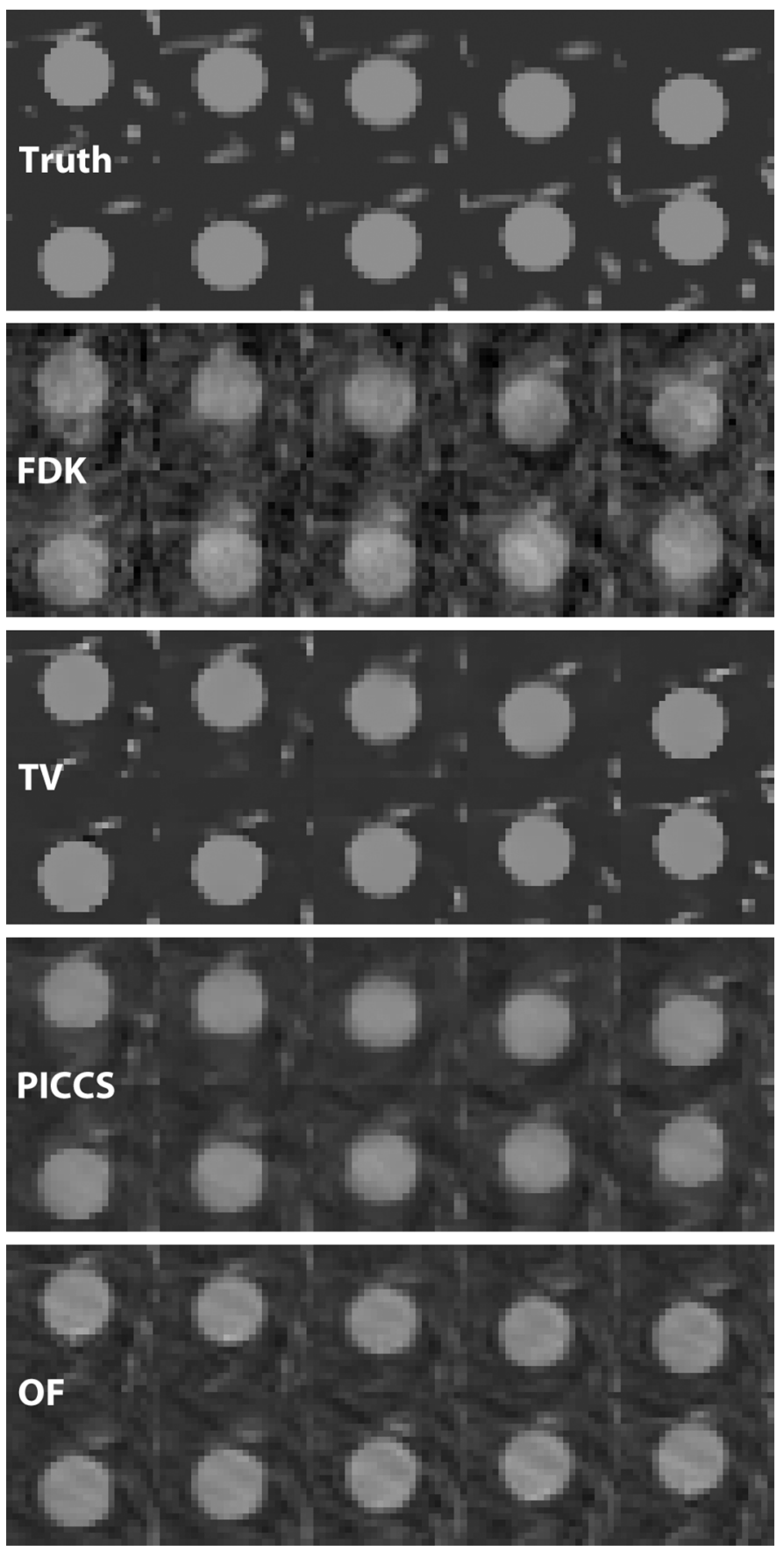

Fig. 4. Tumor definition under respiratory motion in the numerical phantom. 10 respiratory phases are depicted corresponding to one respiratory cycle. The embedding of the anatomical region and applied acronyms were defined in Fig. 3.

motion (Fig. 4) we notice firstly how the total variation term seems to be the best regularizer in the lung. Probably however, TV provides an unrealistically good reconstruction due to its preference towards completely piecewise constant images. This preference is honored by the XCAT phantom but to a lesser degree in-vivo. Another main observation to make from Fig. 4 is the actual delineation of the tumor. Its borders seem most accurately defined by the TV and OF reconstructions and somewhat more blurred for PICCS. Similar conclusions can be made in the close-up of the sternum in Fig. 5. Arguably OF 

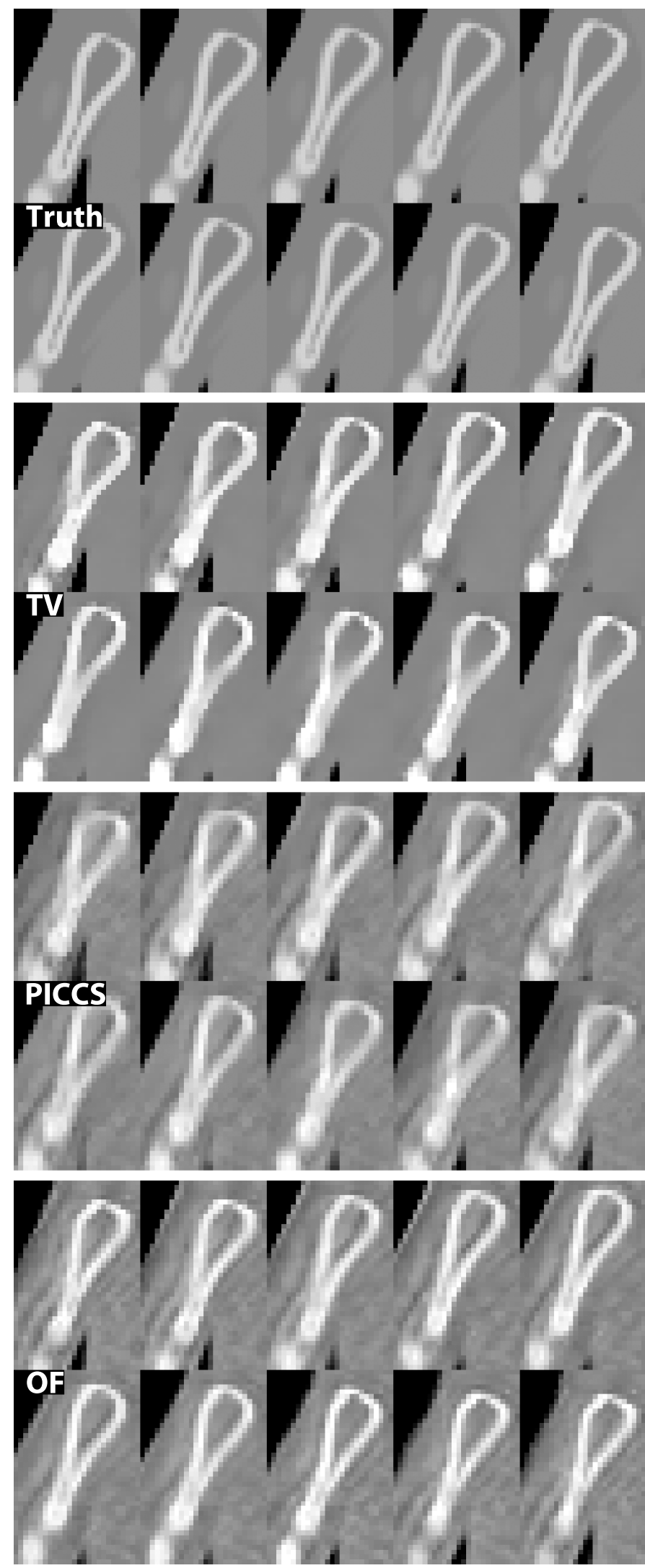

Fig. 5. Sternum definition under respiratory motion. 10 respiratory phases are depicted corresponding to one respiratory cycle. The embedding of the anatomical region and applied acronyms were defined in Fig. 3.
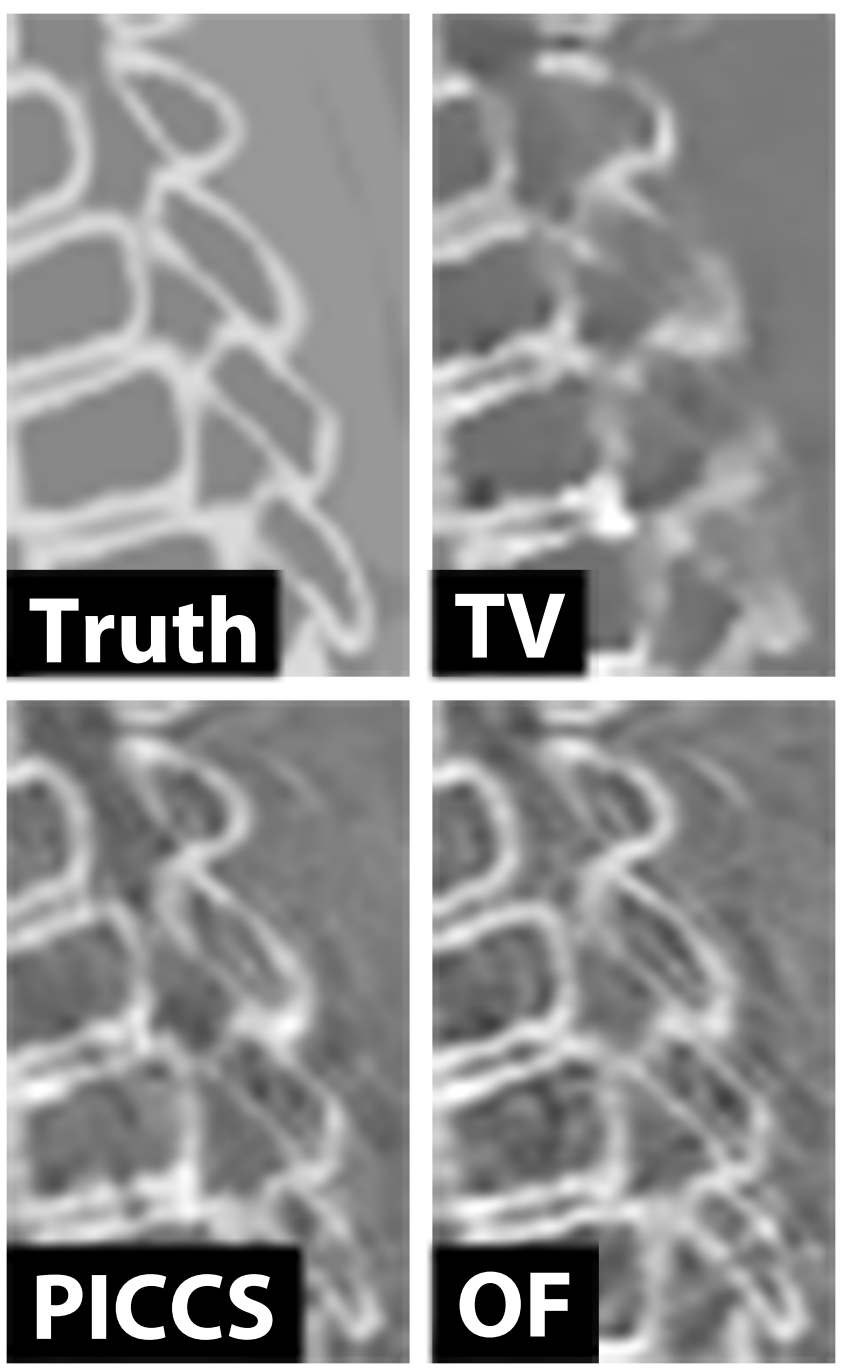

Fig. 6. Definition of stationary part of the spine in the XCAT phantom. The embedding of the anatomical region and applied acronyms were defined in Fig. 3.

offers the better reconstruction over TV when considering the depiction of the bony structures.

We furthermore enlarge a stationary region in the spine for closer inspection (Fig. 6). In this case TV fails to depict the spinous processes whereas both PICCS and OF provide good definition.

A number of measures can be explored in order to quantitatively support our qualitative evaluation results that 1) PICCS results in noticeable more temporal blurring compared to TV and OF, and 2) PICCS and OF offer better definition of the smaller, stationary structures compared to TV. The following metrics are based on the full field of view apart from the extreme top and bottom (see Fig. 3 for a visual illustration as to why the volumes were slightly cropped). A simple metric to evaluate is the peak signal-to-noise errors averaged across the temporal phases. In order of increasing error they are: 1) TV. 31.1; 2) PICCS. 29.4; 3) OF. 29.2; 4) FDK. 21.9. It has been recognized in the past however that the human visual system is adapted to structural image content and that our 


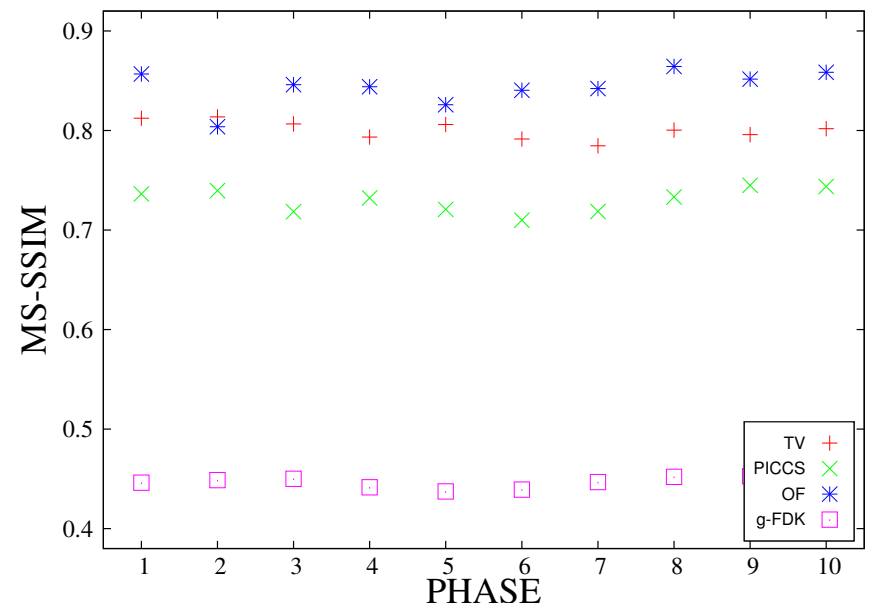

Fig. 7. Multi-scale structural similarity of the g-FDK, TV, PICCS, and OF reconstructions respectively (Fig. 3) to the ground truth. The similarity is plotted for each of 10 temporal phases. The maximally attainable similarity of two images is 1 .

quantitative measures should reflect this [39]. Generalizing the multi-scale structural similarity index introduced in reference [39] to three dimensions, Fig. 7 plots the structural similarity for each temporal phase for the various reconstructions vs. the ground truth. In this metric the proposed registrationbased reconstruction performs consistently better than the alternatives. Taking the average measures over all phases provides the ranking: 1) OF. 0.84; 2) TV. 0.80; 3) PICCS. 0.73 ; 4) g-FDK. 0.45 .

As a measure of reconstruction resolution, i.e. the degree of image blurring, we utilize a method proposed for evaluation of super-resolution MRI [40]: A straight line path from the background to an homogeneous anatomical region is initially defined. A sigmoid is subsequently fitted to the observed intensities along this path. One of the free parameters of the sigmoid is inversely proportional to what we here define as the perceived degree of blurring. An example of a sigmoid fitting is shown in Fig. 8 corresponding to a line entering the tumor enlarged in Fig. 4 from above, i.e. along the direction of motion. The corresponding blurring measures (the lower the number the sharper the edge) are in this example: 1) TV. 2.123 ; 2) OF. 2.470; 3) PICCS. 3.223. A sigmoid fit was computed for each of the 10 respiratory phases for the extent of the tumor ( 9 coronal slices). A boxplot depicting the median and 25th/75th percentiles respectively of the corresponding measures is shown in Fig. 9. Consistently, and in accordance with the qualitative evaluation, the boxplot shows the PICCS reconstructions as having less sharply defined edges compared to the TV and OF reconstructions.

We used a circular Hough transform to quantitatively measure whether the depicted tumor motion was over- or underestimated (according to the segmentation algorithm); The tumor was automatically segmented from a fixed twodimensional region of interest in a coronal imaging slice depicting the tumor in all temporal phases (using Matlab's 'imfindcircles' function). Recall that by construction of the numerical phantom, the tumor underwent respiratory motion

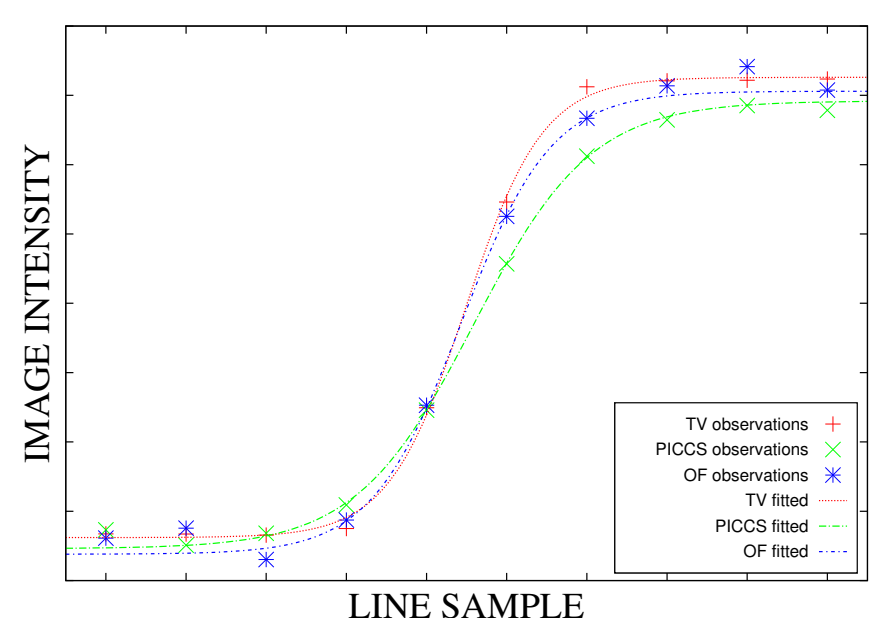

Fig. 8. Sigmoid fitting of intensity profiles. Image intensities are plotted evenly spaced along a straight line from the background entering the tumor (as depicted in Fig. 4). A sigmoid is fitted to each set of observations corresponding to TV, PICCS, and OF reconstructions respectively.

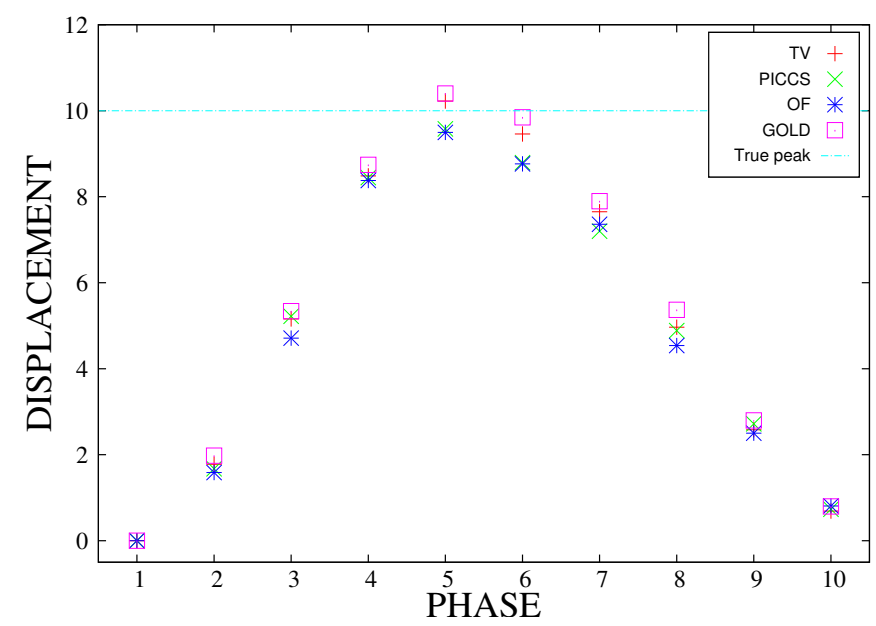

Fig. 10. Tumor motion quantified by a circular Hough transform. The detected feet-head motion is plotted as a function of the temporal phase. The stippled horizontal line indicates the level of the true peak magnitude of the motion. The displacements are plotted in millimeters.

with $10 \mathrm{~mm}$ peak-to-peak amplitude in the feet-head direction. The segmentation was performed on the ground truth images as well as the different reconstructions depicted in Fig. 3. The feet-head displacements of the center points of the detected circles are depicted in Fig. 10. On the ground truth images and TV reconstruction, the segmentation algorithm over-estimated the peak magnitude of tumor motion by $0.4 \mathrm{~mm}$ and $0.2 \mathrm{~mm}$ respectively. Segmentation based on the PICCS and OF reconstructions underestimated the motion with $0.4 \mathrm{~mm}$ and 0.5 $\mathrm{mm}$ respectively. All reconstructions thus achieved sub-voxel precision since the data were reconstructed at a resolution of $1.75 \mathrm{~mm}^{3}$.

Finally we demonstrate that the proposed methodology can also be carried out in practice through reconstructions of a clinical dataset. Fig. 11 shows a coronal and sagittal imaging plane from a single temporal phase. A tumor in the right lung is marked by the white rectangle in the coronal view. The sagittal imaging plane also clearly depicts the tumor. Enlarged 


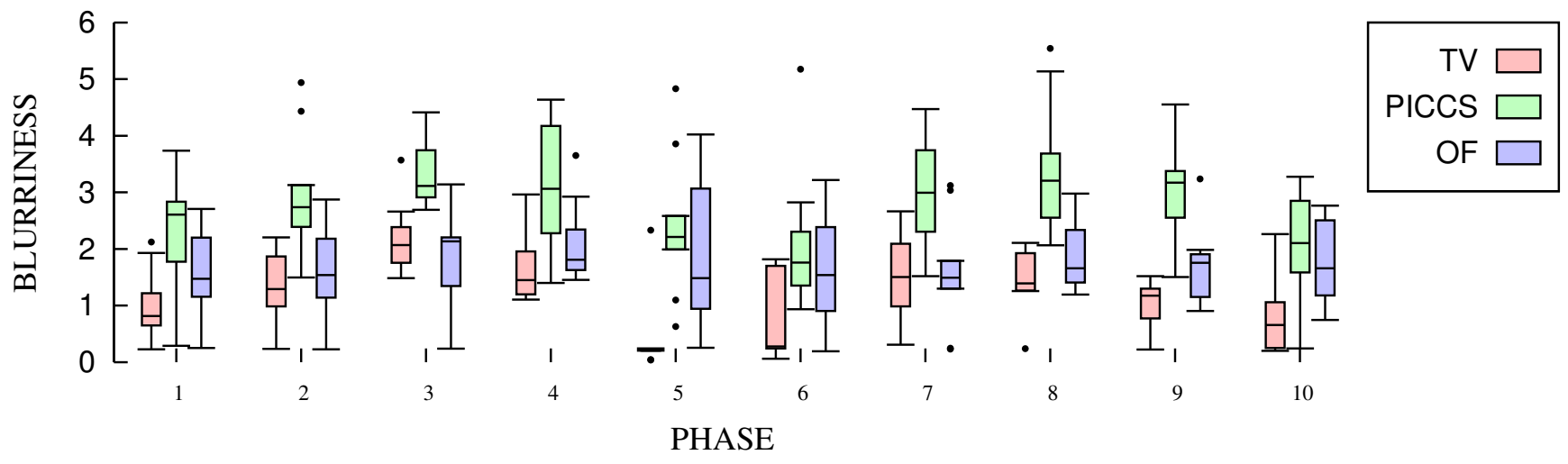

Fig. 9. Boxplot of the median and 25th/75th percentiles of the "blurriness" of edges measured by the sigmoid fit of Fig. 8 corresponding to the XCAT reconstruction in Fig. 4. Higher values indicate flat, blurry edges. The measure approaches 0 as the edge becomes steeper. For each of 10 temporal phases the edge slope is depicted for TV (red), PICCS (green), and OF (blue).
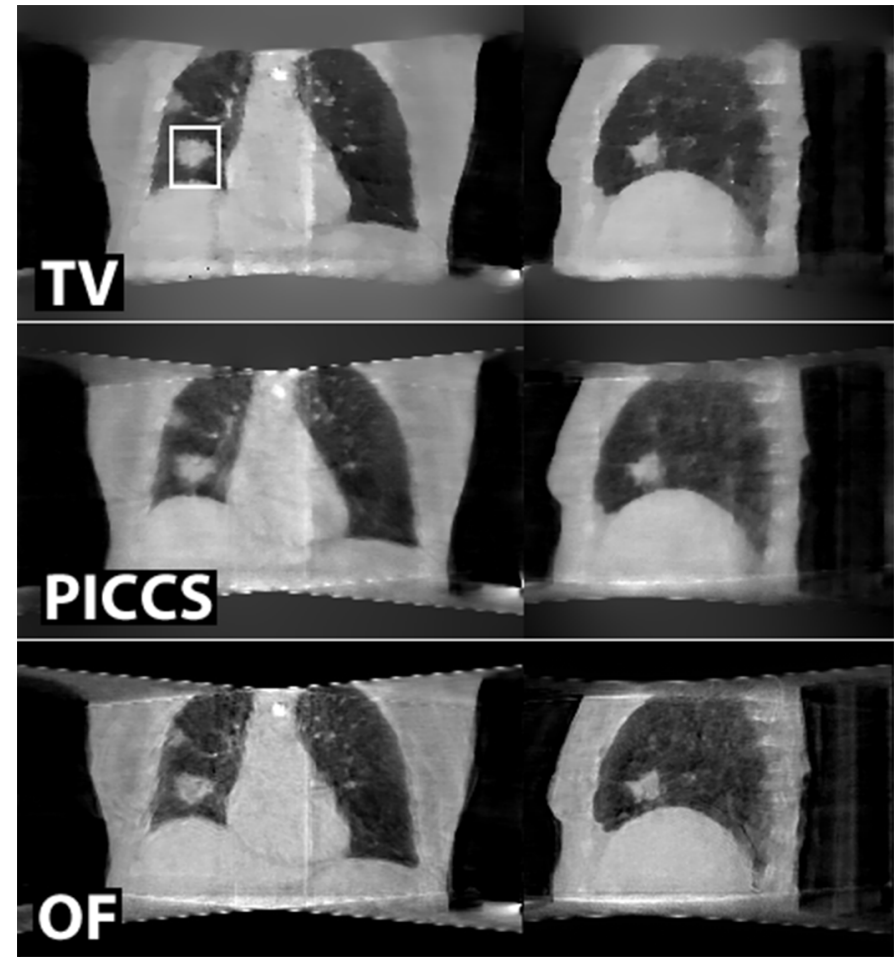

Fig. 11. Reconstruction of clinical dataset. A tumor is visualized in right lung. Total variation regularization (TV), prior image constrained compressed sensing (PICCS), reconstruction based on optical flow inter-phase registration (OF). The square indicates the region enlarged in Fig. 12.

visualizations hereof are provided in Fig. 12. In accordance with the observations made on the phantom reconstructions, the PICCS reconstruction exhibits significant temporal blurring and uncertain tumor delineation when compared to the TV and OF reconstructions. To verify this statement a sigmoid fit was computed for each of the 10 respiratory phases for 10 coronal slices covering the tumor. A boxplot depicting the median and $25 \mathrm{th} / 75$ th percentiles respectively of the corresponding measures is shown in Fig. 13.
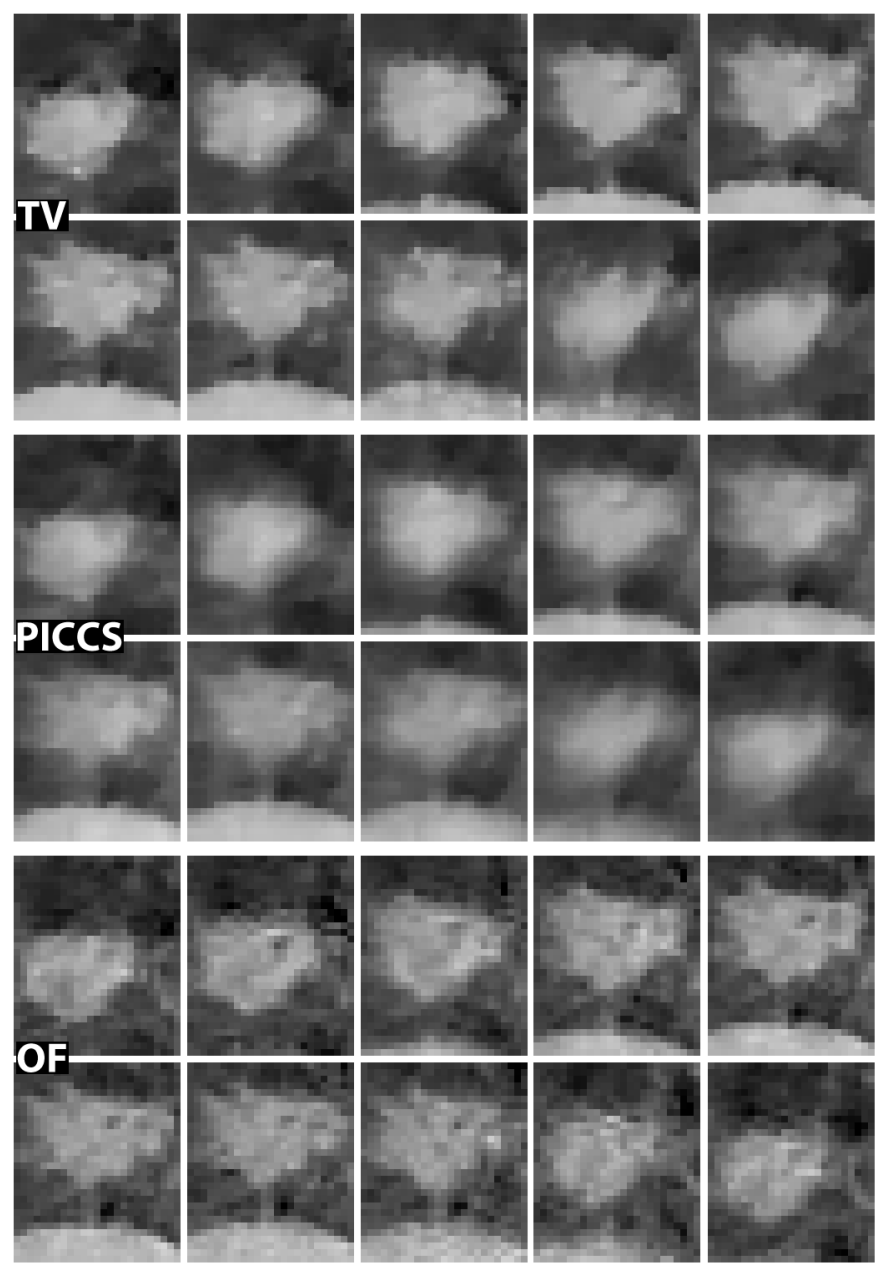

Fig. 12. Tumor definition under respiratory motion in a clinical dataset. 10 respiratory phases are depicted corresponding to one respiratory cycle. The embedding of the anatomical region and applied acronyms were defined in Fig. 11. As a reference to the degree of motion present, the voxel size was reconstructed to $1.75^{3} \mathrm{~mm}^{3}$ and each enlarged section thus corresponds to roughly $5 \mathrm{~cm}$.

\section{Discussion}

A new reconstruction approach for time-resolved CBCT of the thorax was introduced. The main innovation was to 


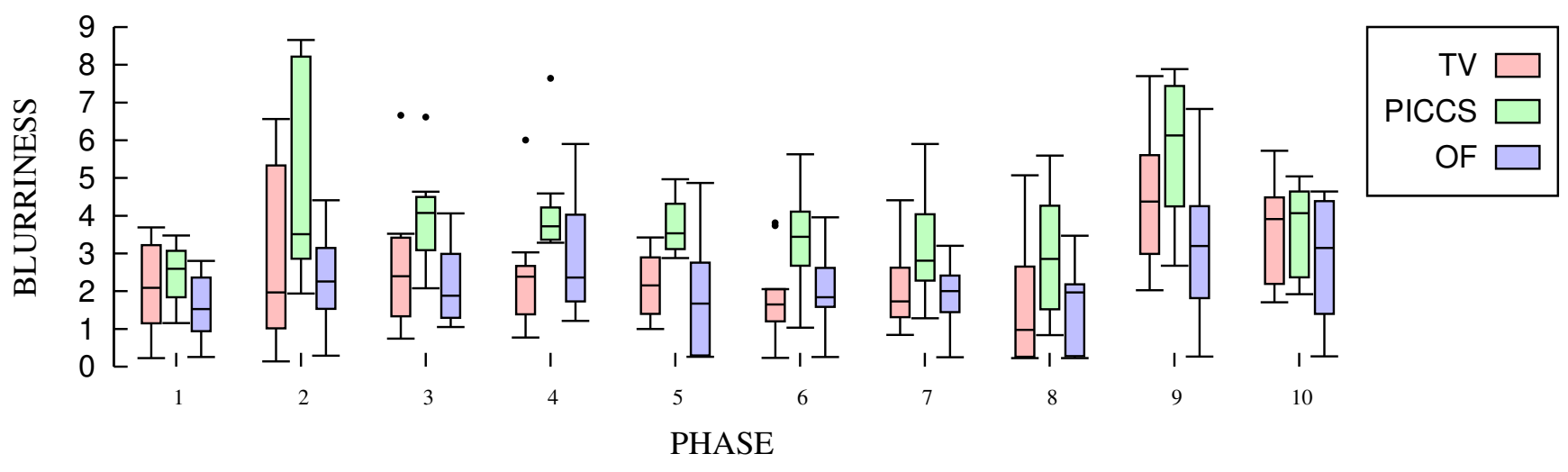

Fig. 13. Boxplot of the median and 25th/75th percentiles of the "blurriness" of edges measured by the sigmoid fit of Fig. 8 corresponding to the clinical reconstruction in Fig. 12. Higher values indicate flat, blurry edges. The measure approaches 0 as the edge becomes steeper. For each of 10 temporal phases the edge slope is depicted for TV (red), PICCS (green), and OF (blue).

utilize image registration to reconstruct every temporal phase from the full set of projections, hereby avoiding significant undersampling in the reconstruction. In the present work we acquired all data from a single flat panel rotation and based our studies hereon. Clinical practice varies however and others are likely to have different preferences, e.g. acquiring less/more data - possibly from several panel revolutions. Certainly, the more projections that are acquired, and the more projections can be assigned to different temporal bins, the higher our expectations of the resulting image quality should be. We believe that the results presented provide a useful overview of the image quality to be expected from registration-based reconstructions under typical clinical constraints.

Conventional reconstruction techniques such as the g-FDK fail for $4 \mathrm{D} \mathrm{CBCT}$ as they are unable to handle the undersampling resulting from temporal binning. State-of-the-art reconstruction algorithms such as TV and PICCS address this problem using compressed sensing. They each have their strong and weak sides. The downside of total variation regularization is that it tends to produce unnaturally patchy (piecewise constant) images and that it can remove fine structures such as the spinous processes as demonstrated in Fig. 6. For PICCS stationary structures remain visible due to the inclusion of a fully sampled temporal average. This comes at the price of some temporal blurring however. TV on the other hand does not suffer from temporal blurring. Both the qualitative and quantitative studies in this paper suggest that reconstruction based on optical flow inter-phase registration offer the better overall compromise: OF is competitive with TV and clearly beats PICCS in the depiction of moving structures (qualitatively shown in Fig. 4, Fig. 5, and Fig. 12 and quantitatively verified in Fig. 7 and Fig. 9). On the other hand, both PICCS and OF outperform TV for thin stationary structures such as the spinous processes in Fig. 6.

Looking at the close-ups of the XCAT reconstructions of the tumor and sternum in Fig. 4 and Fig. 5 respectively, we can estimate the influence of intra-phase motion on the reconstruction quality. Evidently the temporal phases undergoing the most motion (mid-inspiration and mid-expiration) appear somewhat more blurred as the remaining reconstruc- tions. This trend is particularly true for the TV and PICCS reconstructions. The observation is backed up quantitatively in the boxplot in Fig. 9, in which the median values corresponding to the $\mathrm{OF}$ reconstructions show less inter-phase variation compared to TV and PICCS. The picture is less clear for the reconstruction of the patient dataset (Fig. 12) and corresponding boxplot (Fig. 13). This is most likely an indication of inconsistent binning of the raw projection data.

The differences between the various reconstructions were more prominent on the numerical phantom when compared to the clinical dataset. Numerous factors are at play. Firstly, an optimal temporal binning could be performed for the numerical phantom. The binning for the clinical dataset on the other hand is only valid under the assumption of a fully regular breathing pattern and is unlikely fully satisfied in practice. Secondly, the slight angle dependent misalignment of the clinical imager was only corrected with an approximate sinusoidal calibration [41], which may have caused a slight loss of resolution. Finally, we did not perform any scatter correction on the clinical data. While the two latter points may be improved upon in obvious extensions of our current implementation, the first point remains a serious issue for all reconstructions that rely on temporal binning.

The OF reconstruction process involves multiple steps. This is a potential disadvantage. Moreover, it requires a successful registration to be feasible. The optical flow solver we utilize (detailed in [33]) is unable to correctly register 4D g-FDK images due to the present aliasing. This led us to describe a number of intermediate steps producing an approximate TV reconstruction suitable for OF estimation. It was outside the scope of this paper to explore and validate the registration quality obtainable from different intermediate image reconstruction and registration algorithms - and to relate potential registration imprecisions to the quality of the final $\mathrm{OF}$ reconstruction outcome. We did however notice a slightly better image quality in the final $\mathrm{OF}$ reconstructions (particularly for blood vessels in the lung) if the registration was based on the TV reconstructions presented in Fig. 3 and Fig. 11 instead of the reduced quality intermediate TV images utilized in the present work. We have chosen however 
to disclose the reconstructions with the shorter reconstruction time and thus currently the most likely candidate to be useful in clinical practice. We could however also view our approach as a "post-processing step" to any existing 4D reconstruction method; Take as input the 4D reconstruction, perform interphase registration, and utilize the obtained reconstruction to perform a fully sampled final reconstruction. If the registration is valid, the final $4 \mathrm{D}$ reconstruction suppresses undersampling artifacts assuming that sufficient data for a conventional 3D reconstruction was obtained.

A good measure of the expected runtime is the number of multiplications with $E$ and $E^{T}$ respectively that is performed by the solvers. For PICCS and TV we ran 300 Split-Bregman iterations each containing 8 conjugate gradient iterations. Each conjugate gradient iteration contains two operations involving multiplication with $E$ or $E^{T}$. This is supplemented by two additional operations for every Split-Bregman iteration. In total $2 *(2400+300)=5400$ applications of the system matrix was computed during the reconstruction of the presented TV and PICCS reconstructions. For the intermediate TV reconstructions intended for image registration on the other hand, we ran 100 iterations Split-Bregman iterations containing a single conjugate gradient iteration each (on a reduced resolution projection plate). This constitutes "merely" 400 system matrix multiplications. The subsequent $\mathrm{OF}$ reconstruction performed 50 iterations of a gradient projection solver for each of 10 temporal phases. This involved 1000 system matrix multiplications. That is, nearly four times as much work went into the presented TV/PICCS reconstructions compared to the work put into the OF reconstructions. With the present implementation and hardware as stated in section II-G the approximate reconstruction times of a full $\mathrm{OF}$ reconstruction, approximately speaking, constitute 6 hours. Many performance optimizations are however pending and we believe reconstruction times can be reduced noticeable, e.g. by adopting an optimized encoding operator implementation [42]. Naturally, the runtime cost is also defined by the chosen spatial and temporal resolution.

Apart from the initial low-resolution reconstruction we reconstructed at an isotropic resolution of $1.75^{3} \mathrm{~mm}^{3}$ /voxel at 10 temporal phases. This was a compromise made from several considerations: There is no fundamental hindrance to reconstructing at a higher resolution. This would however induce increased signal undersampling and resultant aliasing. Moreover, in our current model it is a requirement that the spatial field of view fully encompasses any voxel intersected from the X-ray source to any detector pixel at any angulation. Thus, as we increase the spatial resolution, memory as well as as computational requirements increase for both the encoding and regularization operators.

An increase in temporal resolution could be made without incurring an extra computational cost to the encoding operator. More registrations would be needed however (scaling quadratically to the number of temporal phases). Since there is a strong correlation between consecutive projections, which typically get binned in series of 3-4 projections, the price in term of aliasing of increasing the temporal resolution may not be very significant. It was considered out of scope in the present work to perform this exploration however.
The low spatial resolution of the training data reconstruction for the principal component analysis was chosen such that, approximately, each bin would be fully sampled and thus exhibit limited temporal aliasing. The PCA on the lowresolution reconstruction was utilized both for the numerical phantom and the clinical datasets to achieve a set of patientspecific PCA basis functions. These were subsequently used as a means of achieving faster convergence of the SplitBregman solver by regularization. As the reconstruction of the low-resolution volume is fast and the PCA computation time negligible, the PCA-based regularization term offers a significant overall reduction in computation time.

The long reconstruction times for 4D CBCT using TV, PICCS or OF is a practical hurdle for clinical applications in which online reconstruction is required. For a number of scenarios however, reconstruction times in the order of a few hours would be acceptable. Radiotherapy treatment planning and evaluation in which a patient is treated daily over a month long course is one such example. 4D CBCT images could be prepared from one day to the next. In fact, the underlying optical flow field in itself could have its applications in e.g. tumor tracking and dose accumulation.

\section{APPENDIX A \\ SPLIT-BREGMAN AND CONJUGATE GRADIENT ALGORITHMS}

This appendix provides implementation details on the constrained Split-Bregman solver. It was used to minimize the $l 1$-regularized optimization problems (3) and (7), i.e. computing the low-resolution training data and the intermediate reconstruction. It was also used to obtain the reference reconstructions for TV and PICCS.

Pseudo-code for the algorithm is provided in Algorithm 1 (adapted from references [27], [28]). E denotes the CBCT operator, $\nabla$ denotes spatial and temporal partial derivative operators according to the subscript, and $\mathbf{A}_{l}$ denotes the PCA operator computed in section II-B. $\lambda, \mu$, and $\gamma$ are user defined regularization weights. Vectors subscripted by the spatio-temporal dimensions relate to the minimization of the total variation according to definitions (6) and (8). Vectors denoted by subscript $P$ relate the minimization of the PCA regularization term, while vectors with subscript ${ }_{N}$ relate to the enforcement of the non-negativity constraint.

A few adaptions were required for the different scenarios; 1) for the low-resolution and TV reconstructions no PCA term was included, 2) for the low-resolution reconstruction no temporal TV regularization was applied, and 3) for PICCS an additional TV term (not shown) was included to regularize by a temporally averaged reconstruction ( $\alpha=0.5$, see reference [11]). The numerical constants $\lambda, \mu$, and $\gamma$ influence the convergence rate, however not the theoretical solution to equations (3) and (7). Numerically however there is a certain range for these constants within which the algorithm remains stable. In the present work we used $\lambda=5.0, \mu=10.0$, and $\gamma=5.0$ on normalized input data. These settings have been used successfully, i.e. with no need for re-tuning, across the datasets we have evaluated so far. 


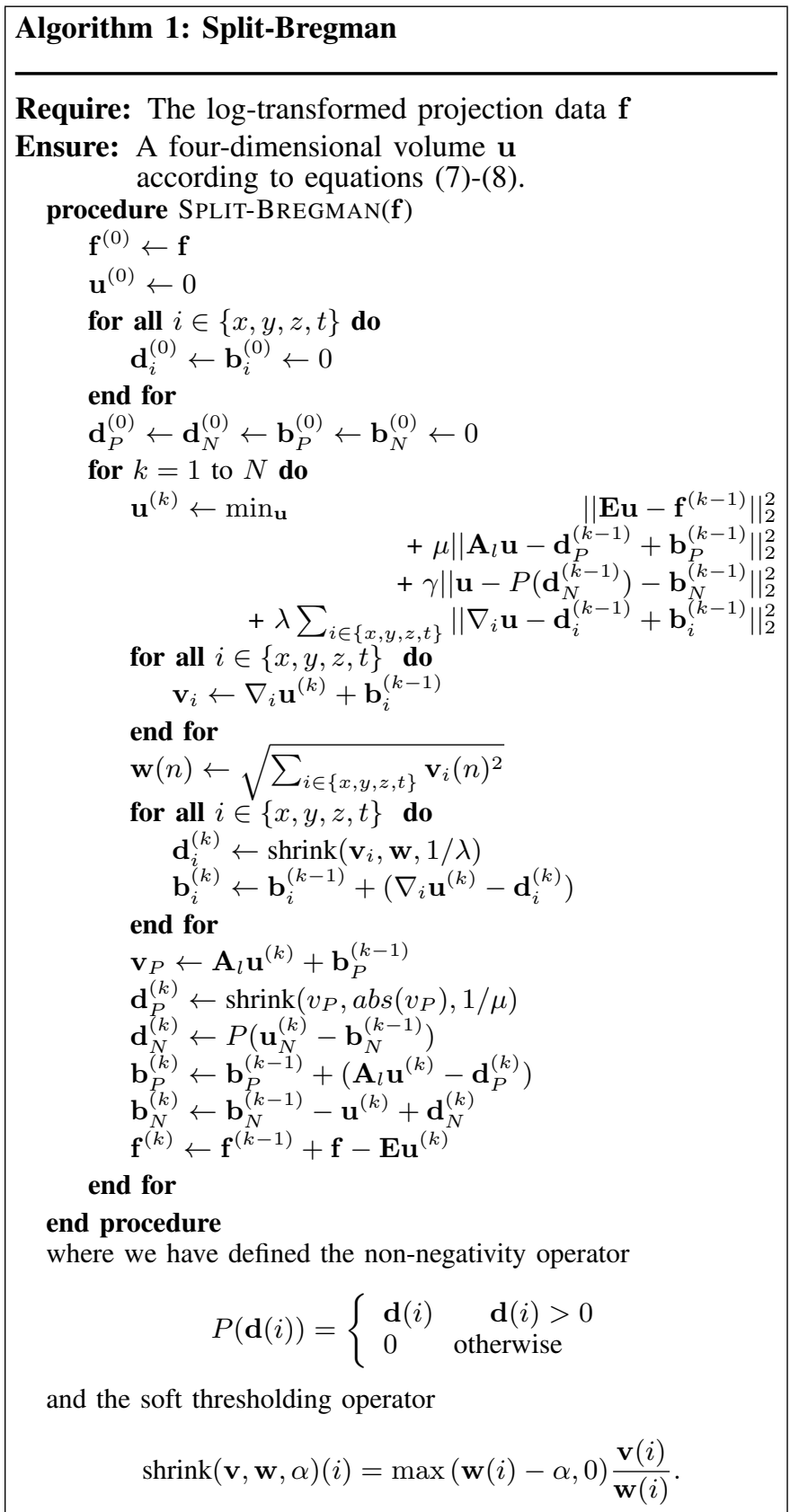

The computational core of the algorithm is the least squares minimization problem in the first line of the loop. The remaining code lines merely define element-wise vector operations. The solution to the given least squares problem is obtained analogously to the derivation of equation (5) from the least squares problem (4). We run a few iterations (as stated in section II-G) of a conjugate gradient solver to approximate the solution. Pseudo-code for the conjugate gradient solver is shown in Algorithm 2.

\section{APPENDIX B \\ OPTICAL FLOW ESTIMATION}

This appendix summarizes the derivation of the threedimensional optical flow algorithm used in the present work. Pseudo-code for the implementation is provided. The method

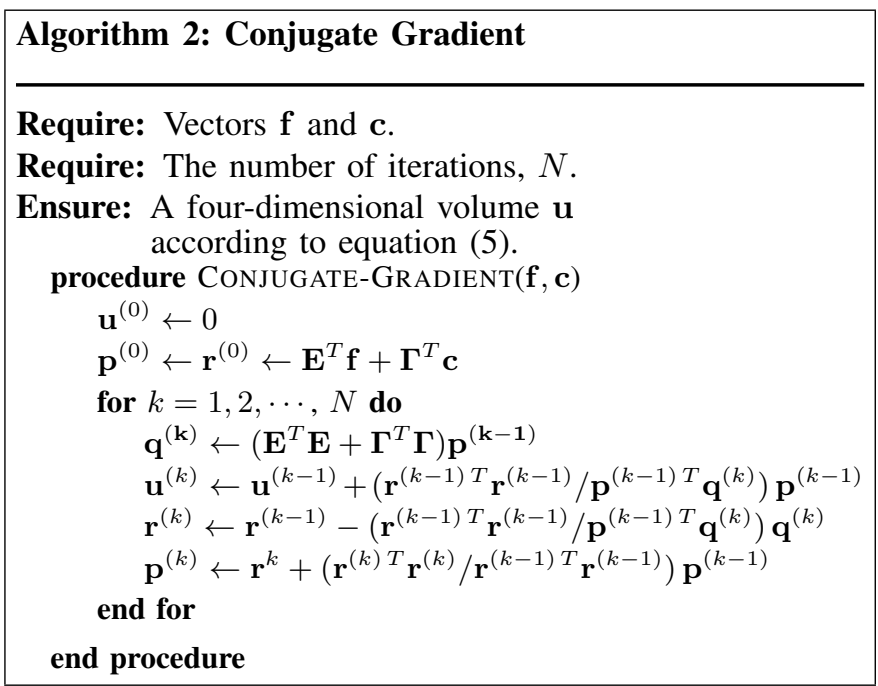

was initially proposed in reference [30] and previously evaluated for both conventional and cone beam computed tomography [32], [33].

Optical flow estimation of an image volume series, $I(x, y, z, t)$, in which $x, y$, and $z$ denotes the spatial dimensions and $t$ denote the temporal dimension, can be stated as finding the vector field $(\delta x, \delta y, \delta z, \delta t)$ such that

$$
I(x, y, z, t)=I(x+\delta x, y+\delta y, z+\delta z, t+\delta t)
$$

Defining $u=\mathrm{d} x / \mathrm{d} t, v=\mathrm{d} y / \mathrm{d} t, w=\mathrm{d} z / \mathrm{d} t, I_{x}=\partial I / \partial x$, $I_{y}=\partial I / \partial y, I_{z}=\partial I / \partial z$, and $I_{t}=\partial I / \partial t$ equation (10) can be approximately described by the well-know aperture problem [30]-[33]:

$$
I_{x} u+I_{y} v+I_{z} w+I_{t}=0 .
$$

Equation (11) states that the material derivatives of the image intensities should be 0 for a valid solution to the registration problem. It was noted in reference [30] that the assumption of complete intensity conservation is unlikely to be met in practice. Consequently the authors proposed to determine yet another unknown variable, $B$, and compute the vector field $(u, v, w, B)$ minimizing for all voxels the data consistency energy $\varepsilon_{b}$ defined as

$$
\mathcal{E}_{b}=B-I_{x} u-I_{y} v-I_{z} w-I_{t} .
$$

Regularization is required to specify an unique solution, thus we minimize instead

$$
\underset{u, v, w, B}{\operatorname{argmin}} \iiint\left(\varepsilon_{b}^{2}+\alpha^{2} \varepsilon_{c}^{2}+\beta^{2} \varepsilon_{I}^{2}\right) \mathrm{d} x \mathrm{~d} y \mathrm{~d} z
$$

where

$$
\mathcal{E}_{c}^{2}=\|\nabla u\|^{2}+\|\nabla v\|^{2}+\|\nabla w\|^{2}, \mathcal{E}_{I}^{2}=\|\nabla B\|^{2},
$$

and $\alpha, \beta$ are user defined regularization weights.

The displacement vector field corresponding to equation (12) is derived from variational calculus, i.e. the EulerLagrange equations. We refer to reference [32] for the full derivation, providing here the solution following an iterative Jacobi update scheme: 


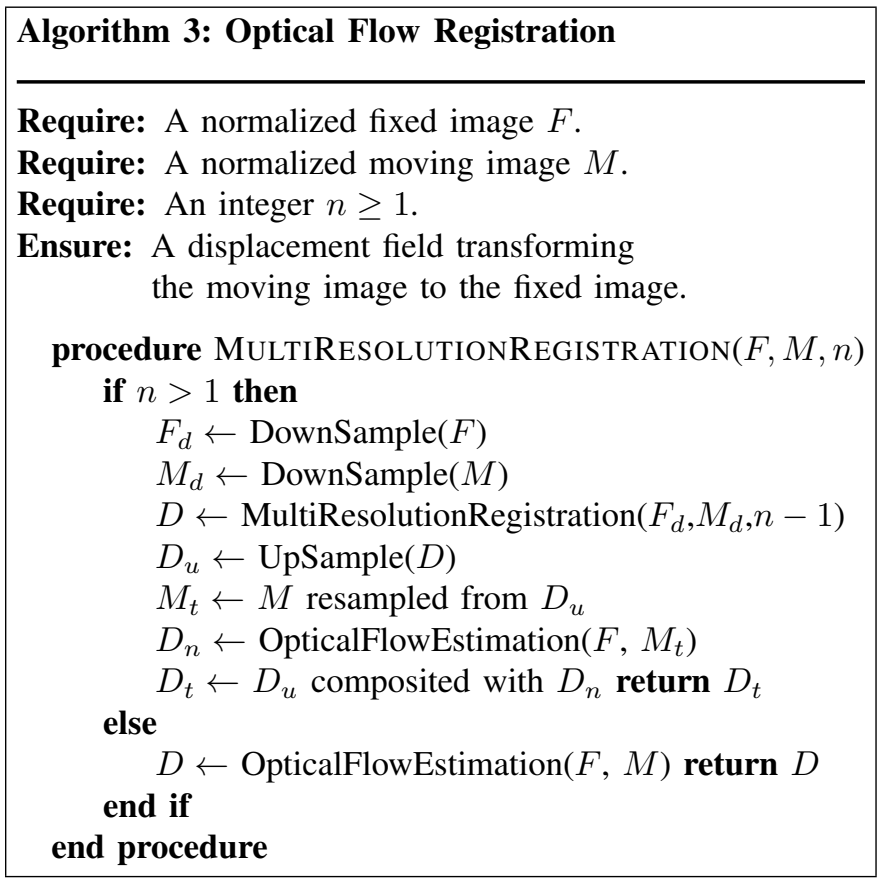

$u^{(k+1)}=\bar{u}^{(k)}-\beta^{2} I_{x}\left(I_{x} \bar{u}^{(k)}+I_{y} \bar{v}^{(k)}+I_{z} \bar{w}^{(k)}+I_{t}-\bar{B}^{(k)}\right) / C$ $v^{(k+1)}=\bar{v}^{(k)}-\beta^{2} I_{y}\left(I_{x} \bar{u}^{(k)}+I_{y} \bar{v}^{(k)}+I_{z} \bar{w}^{(k)}+I_{t}-\bar{B}^{(k)}\right) / C$ $w^{(k+1)}=\bar{w}^{(k)}-\beta^{2} I_{z}\left(I_{x} \bar{u}^{(k)}+I_{y} \bar{v}^{(k)}+I_{z} \bar{w}^{(k)}+I_{t}-\bar{B}^{(k)}\right) / C$ $B^{(k+1)}=\bar{B}^{(k)}+\alpha^{2}\left(I_{x} \bar{u}^{(k)}+I_{y} \bar{v}^{(k)}+I_{z} \bar{w}^{(k)}+I_{t}-\bar{B}^{(k)}\right) / C$ where $\bar{u}, \bar{v}, \bar{w}$, and $\bar{B}$ denote the means of $u, v, w$, and $B$ respectively in a $3 \times 3 \times 3$ neighborhood (excluding the voxel itself), and $C=\beta^{2} I_{x}^{2}+\beta^{2} I_{y}^{2}+\beta^{2} I_{z}^{2}+\alpha^{2}+\alpha^{2} \beta^{2}$.

We take a multi-resolution approach for which pseudocode is provided in Algorithm 3. The two code statements "OpticalFlowEstimation $(F, M)$ " denote the iterative computation of $u, v, w$, and $B$ according to the previously stated formulae; we iterate until no individual spatial displacement vector changes more than 0.01 voxel between consecutive iterations. The two regularization constants were set to $\alpha=0.05$ and $\beta=2.0$ in the present work. Trilinear interpolation was used to resample intra-voxel intensities in the moving image $M$ according to the displacement field.

\section{APPENDIX C \\ GRADIENT PROJECTION ALGORITHM}

This appendix contains pseudo-code for the gradient projection algorithm applied to equation (9) (adapted from [34], [35]). The algorithm is depicted in Algorithm 4 where $\mathbf{E}_{t}$ and $\mathbf{E}_{t^{\prime}}$ denote the CBCT operator for temporal phase $t$ and the remaining phases $t^{\prime}$ respectively, $\mathbf{R}_{t}$ is the resampling operator from temporal phase $t$ to the remaining phases, and $\lambda_{t^{\prime}}$ is the regularization weight.

The algorithm reconstructs one temporal phase $(t)$ and thus should be repeated for all temporal phases. Note that (9) corresponds to solving a linear least squares system. The solver however is non-linear as it enforces a non-negativity constraint. It is straightforward to add total variation regularization to

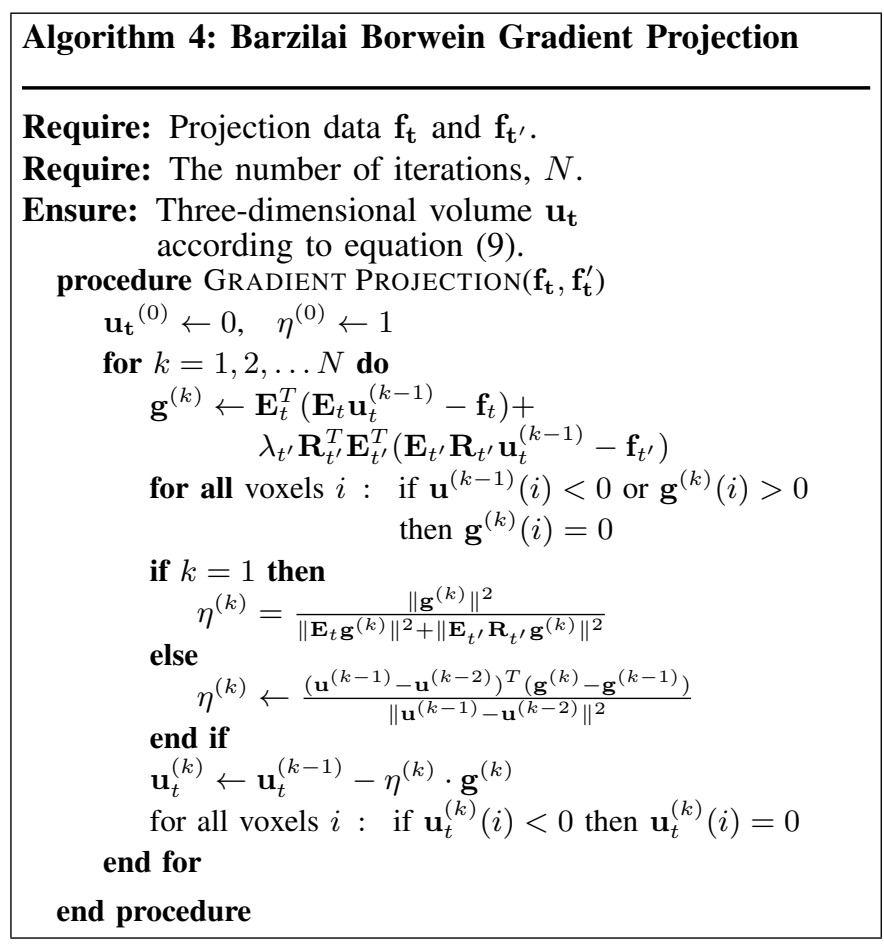

the solver if desired. One merely adapts the line updating $g^{(k)}$ by adding the (weighted) gradient of the desired TV term. Increasing the weighing of such a TV term would gradually transform the outcome of the algorithm towards a piecewise-constant appearance as we see for conventional TV reconstructions.

We varied the regularization weight $\left(\lambda_{t^{\prime}}\right)$ according to the distance between the temporal phases using a Gaussian. I.e. neighboring temporal phases were weighted by 0.90 , and the temporal phase furthest distance from $t$ were weighted by 0.08 .

\section{ACKNOWLEDGMENT}

The authors would like to thank Dr. Henrik Pedersen for discussions on principal component analysis, Dr. Carsten Brink for discussions on registration-based reconstruction, Dr. Michael Schacht Hansen for discussions on the implementation of the present work inside the Gadgetron framework, and Dr. Ole Østerby for discussions of various math issues. This research was financially supported by CIRRO - The Lundbeck Foundation Center for Interventional Research in Radiation Oncology and The Danish Council for Strategic Research.

\section{REFERENCES}

[1] D. A. Jaffray, J. H. Siewerdsen, J. W. Wong, and A. A. Martinez, "Flatpanel cone-beam computed tomography for image-guided radiation therapy," Int. J. Radiat. Oncol. Biol. Phys., vol. 53, no. 5, pp. 13371349, Aug 2002.

[2] R. C. Orth, M. J. Wallace, and M. D. Kuo, "C-arm cone-beam CT: general principles and technical considerations for use in interventional radiology," J Vasc Interv Radiol, vol. 19, no. 6, pp. 814-820, Jun 2008.

[3] R. Ning, B. Chen, R. Yu, D. Conover, X. Tang, and Y. Ning, "Flat panel detector-based cone-beam volume CT angiography imaging: system evaluation," IEEE Trans Med Imaging, vol. 19, no. 9, pp. 949-963, Sep 2000.

[4] L. A. Feldkamp, L. C. Davis, and J. W. Kress, "Practical cone-beam algorithm," JOSA A, vol. 1, no. 6, pp. 612-619, 1984. 
[5] L. Zijp, J. Sonke, and M. van Herk, "Extraction of the Respiratory Signal from Sequential Thorax Cone-Beam X-Ray Images," ICCR 04, Seoul, pp. 507-509, 2004.

[6] S. Rit, M. van Herk, L. Zijp, and J. J. Sonke, "Quantification of the variability of diaphragm motion and implications for treatment margin construction," Int. J. Radiat. Oncol. Biol. Phys., vol. 82, no. 3, pp. 399407, Mar 2012.

[7] A. Kavanagh, P. M. Evans, V. N. Hansen, and S. Webb, "Obtaining breathing patterns from any sequential thoracic x-ray image set," Phys Med Biol, vol. 54, no. 16, pp. 4879-4888, Aug 2009.

[8] I. Vergalasova, J. Cai, and F. F. Yin, "A novel technique for markerless, self-sorted 4D-CBCT: feasibility study," Med Phys, vol. 39, no. 3, pp. 1442-1451, Mar 2012.

[9] G. C. Mc Kinnon and R. H. Bates, "Towards imaging the beating heart usefully with a conventional CT scanner," IEEE Trans Biomed Eng, vol. 28, no. 2, pp. 123-127, Feb 1981.

[10] E. Y. Sidky and X. Pan, "Image reconstruction in circular cone-beam computed tomography by constrained, total-variation minimization," Phys Med Biol, vol. 53, no. 17, pp. 4777-4807, Sep 2008.

[11] S. Leng, J. Tang, J. Zambelli, B. Nett, R. Tolakanahalli, and G. H. Chen, "High temporal resolution and streak-free four-dimensional cone-beam computed tomography," Phys Med Biol, vol. 53, no. 20, pp. 5653-5673, Oct 2008.

[12] G. H. Chen, P. Theriault-Lauzier, J. Tang, B. Nett, S. Leng, J. Zambelli, Z. Qi, N. Bevins, A. Raval, S. Reeder, and H. Rowley, "Time-resolved interventional cardiac C-arm cone-beam CT: an application of the PICCS algorithm," IEEE Trans Med Imaging, vol. 31, no. 4, pp. 907923, Apr 2012.

[13] T. Li, E. Schreibmann, Y. Yang, and L. Xing, "Motion correction for improved target localization with on-board cone-beam computed tomography," Phys Med Biol, vol. 51, no. 2, pp. 253-267, Jan 2006.

[14] D. Sarrut, V. Boldea, S. Miguet, and C. Ginestet, "Simulation of fourdimensional CT images from deformable registration between inhale and exhale breath-hold CT scans," Med Phys, vol. 33, no. 3, pp. 605-617, Mar 2006.

[15] J. Ehrhardt, R. Werner, D. Saring, T. Frenzel, W. Lu, D. Low, and H. Handels, "An optical flow based method for improved reconstruction of 4D CT data sets acquired during free breathing," Med Phys, vol. 34, no. 2, pp. 711-721, Feb 2007.

[16] T. Berkus, M. Oehlhafen, P. Kunz, and M. Kachelriess, "Motion correction for improved target localization with on-board cone-beam computed tomography," IEEE Nuclear Science Symposium and Medical Imaging Conference, pp. 3986-3993, Oct 2011.

[17] M. Brehm, P. Paysan, M. Oelhafen, P. Kunz, and M. Kachelriess, "Selfadapting cyclic registration for motion-compensated cone-beam CT in image-guided radiation therapy," Med Phys, vol. 39, no. 12, pp. $7603-$ 7618, Dec 2012

[18] J. Hinkle, M. Szegedi, B. Wang, B. Salter, and S. Joshi, "4D CT image reconstruction with diffeomorphic motion model," Med Image Anal, vol. 16, no. 6, pp. 1307-1316, Aug 2012.

[19] B. A. Mair, D. R. Gilland, and J. Sun, "Estimation of images and nonrigid deformations in gated emission CT," IEEE Trans Med Imaging, vol. 25, no. 9, pp. 1130-1144, Sep 2006.

[20] M. Blume, A. Martinez-Moller, A. Keil, N. Navab, and M. Rafecas, "Joint reconstruction of image and motion in gated positron emission tomography," IEEE Trans Med Imaging, vol. 29, no. 11, pp. 1892-1906, Nov 2010.

[21] M. S. Hansen, T. S. Sørensen, A. E. Arai, and P. Kellman, "Retrospective reconstruction of high temporal resolution cine images from real-time MRI using iterative motion correction," Magn Reson Med, vol. 68, no. 3, pp. 741-750, Sep 2012.

[22] F. Rousseau, O. A. Glenn, B. Iordanova, C. Rodriguez-Carranza, D. B Vigneron, J. A. Barkovich, and C. Studholme, "Registration-based approach for reconstruction of high-resolution in utero fetal MR brain images," Acad Radiol, vol. 13, no. 9, pp. 1072-1081, Sep 2006.

[23] H. Jung, K. Sung, K. S. Nayak, E. Y. Kim, and J. C. Ye, "k-t FOCUSS: a general compressed sensing framework for high resolution dynamic MRI," Magn Reson Med, vol. 61, no. 1, pp. 103-116, Jan 2009.

[24] H. Pedersen, S. Kozerke, S. Ringgaard, K. Nehrke, and W. Y. Kim, "k-t PCA: temporally constrained k-t BLAST reconstruction using principal component analysis," Magn Reson Med, vol. 62, no. 3, pp. 706-716, Sep 2009.

[25] H. Gao, J. F. Cai, Z. Shen, and H. Zhao, "Robust principal component analysis-based four-dimensional computed tomography," Phys Med Biol, vol. 56, no. 11, pp. 3181-3198, Jun 2011.
[26] W. P. Segars, G. Sturgeon, S. Mendonca, J. Grimes, and B. M. Tsui, "4D XCAT phantom for multimodality imaging research," Med Phys, vol. 37, no. 9, pp. 4902-4915, Sep 2010.

[27] T. Goldstein and S. Osher, "The split bregman method for 11-regularized problems," SIAM J. Imaging Sciences, vol. 2, no. 2, pp. 323-343, 2009.

[28] A. Szlam, G. Zhaohui, and S. Osher, "A split Bregman method for non-negative sparsity penalized least squares with applications to hyperspectral demixing , 17th IEEE International Conference on Image Processing, pp. 1917-1920, 2010.

[29] D. L. Donoho, "Compressed sensing," IEEE Transactions on Information Theory, vol. 52, no. 4, pp. 1289-1306, 2006

[30] N. Cornelius and T. Kanade, "Adapting optical-flow to measure object motion in reflectance and x-ray image sequences," $A C M S I G$ GRAPH/SIGART interdisciplinary workshop on Motion: representation and perception, pp. 145-153, 1986.

[31] B. K. P. Horn and B. Schunck, "Determining optical flow," Artif. Intell., vol. 17 , no. $1-3$, pp. $185-203,1981$.

[32] K. Ø. Noe, "Deformable Image Registration for Use in Radiotherapy Using GPU Acceleration," Ph.D. dissertation, Aarhus University, Denmark, July 2009.

[33] K. Ø. Noe, B. D. De Senneville, U. V. Elstrøm, K. Tanderup, and T. S. Sørensen, "Acceleration and validation of optical flow based deformable registration for image-guided radiotherapy," Acta Oncol, vol. 47, no. 7, pp. 1286-1293, 2008.

[34] T. L. Jensen, J. H. Jørgensen, P. C. Hansen, and S. H. Jensen, "Implementation of an optimal first-order method for strongly convex total variation regularization," BIT Numerical Mathematics, vol. 52, no. 2, pp. 329-356, June 2012.

[35] J. C. Park, B. Song, J. S. Kim, S. H. Park, H. K. Kim, Z. Liu, T. S. Suh, and W. Y. Song, "Fast compressed sensing-based CBCT reconstruction using Barzilai-Borwein formulation for application to on-line IGRT," Med Phys, vol. 39, no. 3, pp. 1207-1217, Mar 2012.

[36] J. Hsieh, Computed Tomography. Principles, Design, Artifacts, and Recent Advances, 2nd edition. Wiley-Interscience. SPIE Press, 2009.

[37] M. S. Hansen and T. S. Sørensen, "Gadgetron: An open source framework for medical image reconstruction," Magn Reson Med, Jul 2012.

[38] G. Zeng and G. Gullberg, "Unmatched projector/backprojector pairs in an iterative reconstruction algorithm," Medical Imaging, IEEE Transactions on, vol. 19, no. 5, pp. 548-555, may 2000.

[39] Z. Wang, A. C. Bovik, H. R. Sheikh, and E. P. Simoncelli, "Image quality assessment: from error visibility to structural similarity," IEEE Trans Image Process, vol. 13, no. 4, pp. 600-612, Apr 2004.

[40] H. Greenspan, G. Oz, N. Kiryati, and S. Peled, "MRI inter-slice reconstruction using super-resolution," Magn Reson Imaging, vol. 20, no. 5, pp. 437-446, Jun 2002.

[41] B. Cho, P. R. Poulsen, A. Sloutsky, A. Sawant, and P. J. Keall, "First demonstration of combined $\mathrm{kV} / \mathrm{MV}$ image-guided real-time dynamic multileaf-collimator target tracking," Int. J. Radiat. Oncol. Biol. Phys., vol. 74, no. 3, pp. 859-867, Jul 2009.

[42] H. Gao, "Fast parallel algorithms for the x-ray transform and its adjoint," Med Phys, vol. 39, no. 11, pp. 7110-7120, Nov 2012. 